\title{
A MUSCL-TYPE SEGREGATED - EXPLICIT STAGGERED SCHEME FOR THE EULER EQUATIONS
}

\author{
L. GASTALDO *, R. HERBIN †, J.-C. LATCHÉ $\ddagger$, AND N. THERME $\S$
}

June 8, 2018

\begin{abstract}
We present a numerical scheme for the solution of Euler equations based on staggered discretizations and working either on structured schemes or on general simplicial or tetrahedral/hexahedral meshes. The time discretization is performed by a fractional-step or segregated algorithm involving only explicit steps. The scheme solves the internal energy balance, with corrective terms to ensure the correct capture of shocks, and, more generally, the consistency in the Lax-Wendroff sense. To keep the density, the internal energy and the pressure positive, conditionally positivity-preserving convection operators for the mass and internal energy balance equations are designed by a MUSCL-like procedure: first, second-order in space fluxes are computed, then a limiting procedure is applied. This latter is purely algebraic: it does not require any geometric argument and thus works on quite general meshes; moreover, it keeps the pressure constant at contact discontinuities. The construction of the fluxes does not need any Riemann or approximate Riemann solver, and yields thus a particularly simple algorithm. Artificial viscosity is added in order to reduce the oscillations of the scheme. Numerical tests confirm the accuracy of the scheme.
\end{abstract}

Key words. finite volumes, finite elements, staggered discretizations, Euler equations, compressible flows, analysis.

AMS subject classifications. $65 \mathrm{M} 12$

1. Introduction. The main objective of this paper is to develop and test a numerical scheme for the simulation of non viscous compressible flows modeled by the Euler equations for an ideal gas:

$$
\begin{aligned}
& \partial_{t} \rho+\operatorname{div}(\rho \boldsymbol{u})=0, \\
& \partial_{t}(\rho \boldsymbol{u})+\operatorname{div}(\rho \boldsymbol{u} \otimes \boldsymbol{u})+\nabla p=0, \\
& \partial_{t}(\rho E)+\operatorname{div}(\rho E \boldsymbol{u})+\operatorname{div}(p \boldsymbol{u})=0, \\
& p=(\gamma-1) \rho e, \quad E=\frac{1}{2}|\boldsymbol{u}|^{2}+e,
\end{aligned}
$$

where $t$ stands for the time, $\rho, \boldsymbol{u}, p, E$ and $e$ are the density, velocity, pressure, total energy and internal energy respectively, and $\gamma>1$ is a coefficient specific to the considered fluid. The problem is supposed to be posed over $\Omega \times(0, T)$, where $\Omega$ is an open bounded connected subset of $\mathbb{R}^{d}, 1 \leq d \leq 3$, and $(0, T)$ is a finite time interval. System (1.1) is complemented by initial conditions for $\rho, e$ and $\boldsymbol{u}$, denoted by $\rho_{0}, e_{0}$ and $\boldsymbol{u}_{0}$ respectively, with $\rho_{0}>0$ and $e_{0}>0$, and by a boundary condition which we suppose to be $\boldsymbol{u} \cdot \boldsymbol{n}=0$ at any time and a.e. on $\partial \Omega$, where $\boldsymbol{n}$ stands for the normal vector to the boundary.

Let us list here the essential features of the proposed numerical scheme:

- We use a staggered arrangement of the unknowns, on general simplicial or quadrangular/hexahedral meshes. The scalar variables, namely density, pressure and thus, to allow a straightforward formulation of the equation of state, internal energy, are approximated by piecewise constant functions over the cells while the velocity is approximated at the faces of the cells. Two types of discretizations are used: the celebrated MAC scheme [24] on rectangular Cartesian meshes, and a finite volume scheme using the discontinuous linear finite element degrees of freedom on simplices or hexahedra (3D) or quadrangles $(2 \mathrm{D})$; these finite elements are known as Crouzeix-Raviart for simplices, and Rannacher-Turek for hexhahedra or quadrangles, so we shall denote these discretizations by $\mathrm{CR}$ or RT in the sequel.

\footnotetext{
*Institut de Radioprotection et de Sûreté Nucléaire (IRSN) (laura.gastaldo@irsn.fr)

†Université d'Aix-Marseille (herbin@cmi.univ-mrs.fr)

¥Institut de Radioprotection et de Sûreté Nucléaire (IRSN) (jean-claude.latche@irsn.fr)

$\S$ Institut de Radioprotection et de Sûreté Nucléaire (IRSN); present address Université de Nantes (nicolas.therme@univ-nantes.fr)
} 
The MAC scheme involves only the normal component of the velocity on each face (3D) or edge (2D), while the CR/RT schemes demand the approximation of the full velocity on the faces or edges; the latter scheme is therefore more costly but allows for more general meshes.

- Balance equations are solved successively, and each step is explicit, in the sense that apart from the time-derivative, all terms involve only known quantities at this stage and thus do not require any linear system solver; however these quantities are not necessarily beginning-of-step quantities, so that, strictly speaking, the scheme is not explicit for the full system.

- The energy equation solved by the scheme is the internal energy balance, see Equations (3.2)-(3.3) below; this offers two advantages: first of all, it allows to preserve, by construction of the scheme, the positivity of the internal energy; in addition, it avoids building an approximation of the total energy which, for staggered discretizations, is a "composite" variable, in the sense that it combines quantities discretized on the cells and at the faces. Note that a blunt discretization of the internal energy balance is known to yield wrong shock solutions; a corrective term is added here to circumvent this problem.

- The positivity of the scheme, in the sense that the approximate density and internal energy, and thus the pressure, are positive, is obtained by building a positivitypreserving convection operator for both the mass and internal energy balance equations. For a first-order scheme, it consists in using an upwinding with respect to the material velocity only [27]; here, a higher order scheme is sought, and thus a MUSCL-like procedure is developed, see [41] for the original MUSCL scheme: we compute formally second-order in space fluxes and then apply a limiting procedure to obtain positivity under a CFL-like condition, since we use a explicit time discretization. Inspired from the work performed in [34] for the transport equation, this limiting step is purely algebraic: it does not require any geometric argument and thus works on quite general meshes. It is carefully designed to keep the pressure constant in the zones where it actually should be, and in particular across contact discontinuities.

Such a scheme is often referred to in the literature as a "flux splitting scheme", since it may be obtained by

- splitting the system by a two-step technique, usually into a "convective" and "acoustic" part,

- applying a standard scheme to each part; for the convection system, such a first order scheme yields an upwinding with respect to the material velocity;

- summing both steps to obtain the final flux.

Works in this direction may be found in $[38,33,45,32,40]$. Here, strictly following this line seems difficult, since we work on staggered meshes and with a non-conservative formulation of the system, and obtain some non-standard fluxes; in particular, the pressure gradient is discretized as the dual of the velocity divergence, and thus essentially centered. However, the scheme used here presents similarities with the above references, and its derivation does not use the ingredients usual in the context of hyperbolic systems, in particular, Riemann or approximate Riemann solvers, see e.g. [39, 19, 6] for surveys.

- Finally, the limiting procedure introduces a rather low stabilizing viscosity in the scheme: roughly speaking, the numerical viscosity is at most scaled by the material velocity, and this may not be sufficient in the zones where the local Mach number is low. An alternative numerical diffusion for a staggered scheme, obtained thanks to a kinetic approach and which does not vanish with the velocity, may be found in [4]. Here we follow a different line: to cope with this problem, we add in the scheme a non-linear diffusion, in the sense that it depends on the solution, in the spirit of $[21,22,28]$. However, this diffusion is only introduced in the momentum balance equation; indeed, in the present context, its main purpose is to get a better approximation of the (1D) contact discontinuity, where the density and internal 
energy are discontinuous while the velocity is constant, and which is known to be the part of the solution where the scheme diffusion spoils the accuracy of the approximation.

The work presented here is an extension of [27]: indeed, the scheme proposed in [27] is only first-order in space and stabilization through a non-linear viscosity is not implemented; numerical tests are provided to show that the new scheme is much more accurate than its first-order variant; in addition, we observe that the straightforward formulation of the fluxes yields a low cpu-time consuming algorithm since it does not require any Riemann or approximate Riemann solver.

Note that implicit and partially implicited variants of the scheme [27] have been presented in $[25,20]$ under the form of pressure-correction algorithms, for Euler and Navier-Stokes equations respectively, which are shown to be unconditionally stable, i.e. stable irrespectively of the time and space steps. These schemes boil down to usual pressure correction schemes for incompressible flows when the Mach number tends to zero, with inf-sup stable discretizations. Note also that weak (Lax-Wendroff type) consistency results are shown for this class of schemes with a first order approximation [27]. On going work is conducted to prove the weak consistency for the scheme presented here. This paper is organized as follows. The space discretization is

described in Section 2, and the scheme is given in Section 3. Numerical experiments are presented in Section 4.

2. Meshes and unknowns. In this section, we focus on the discretization of a multi-dimensional domain (i.e. $d=2$ or $d=3$ ); the one-dimensional case is easily obtained from the present description.

Let $\mathcal{M}$ be a mesh of the domain $\Omega$, supposed to be regular in the usual sense of the finite element literature, e.g. [11]. The cells of the mesh are chosen according to the geometry of the domain:

- for a general domain $\Omega$, they can be either non-degenerate quadrilaterals $(d=2)$ or hexahedra $(d=3)$, or simplices, both types of cells being possibly combined in a same mesh,

- for a domain whose boundaries are hyperplanes normal to a coordinate axis, they can be chosen to be rectangles $(d=2)$ or rectangular parallelepipeds $(d=3)$, whose faces are then also necessarily normal to a coordinate axis.

By $\mathcal{E}$ and $\mathcal{E}(K)$ we denote the set of all $(d-1)$-faces $\sigma$ of the mesh and of the element $K \in \mathcal{M}$ respectively. The set of faces included in the boundary of $\Omega$ is denoted by $\mathcal{E}_{\text {ext }}$ and the set of internal faces (i.e. $\mathcal{E} \backslash \mathcal{E}_{\text {ext }}$ ) is denoted by $\mathcal{E}_{\text {int }}$; a face $\sigma \in \mathcal{E}_{\text {int }}$ separating the cells $K$ and $L$ is denoted by $\sigma=K \mid L$. The outward normal vector to a face $\sigma$ of $K$ is denoted by $\boldsymbol{n}_{K, \sigma}$. For $K \in \mathcal{M}$ and $\sigma \in \mathcal{E}$, we denote by $|K|$ the measure of $K$ and by $|\sigma|$ the $(d-1)$-measure of the face $\sigma$.

For any cell shape, the degrees of freedom for the pressure, the density and the internal energy (i.e. the discrete pressure, density and internal energy unknowns) are associated to the cells of the mesh $\mathcal{M}$, and are denoted by:

$$
\left\{p_{K}, \rho_{K}, e_{K}, K \in \mathcal{M}\right\} \text {. }
$$

Let us now turn to the degrees of freedom for the velocity (i.e. the discrete velocity unknowns), and their associated cell(s), referred to in the following as the dual cell(s).

- General meshes - The degrees of freedom are the same for all the velocity components and are located at the center of the faces of the mesh, so the set of degrees of freedom reads:

$$
\left\{u_{\sigma, i}, \sigma \in \mathcal{E}, 1 \leq i \leq d\right\} .
$$

In this case, the discretization thus shares the same unknowns as the low-order nonconforming Rannacher-Turek [35] or Crouzeix-Raviart [14] elements, and is referred to in the following as RT and CR discretizations. 


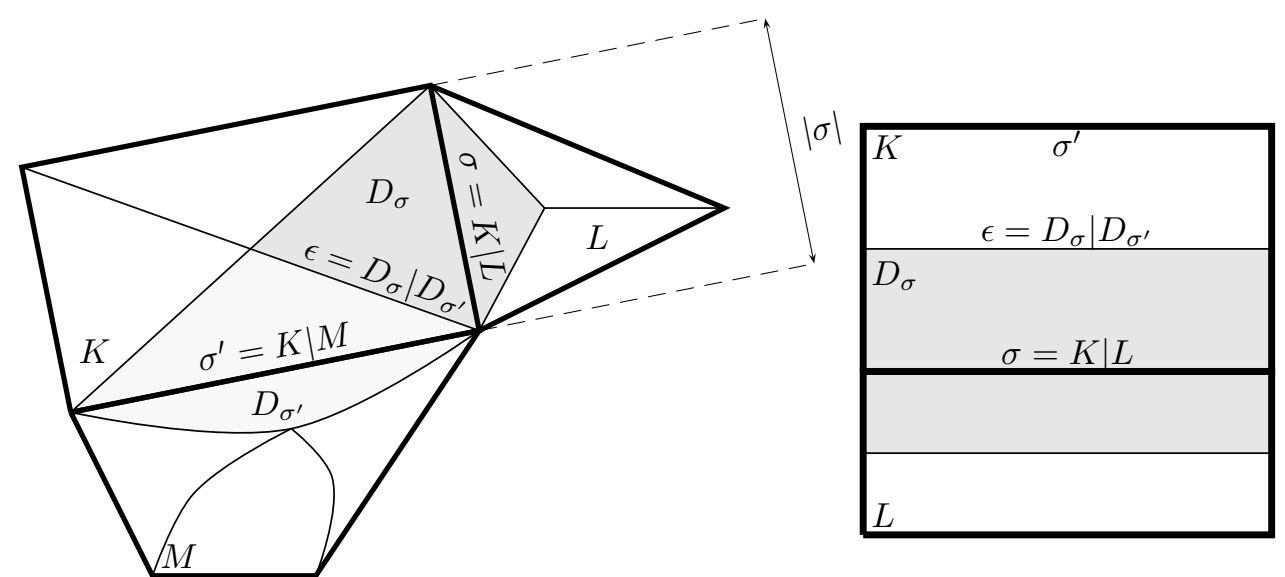

FIG. 2.1. Notations for control volumes and dual cells - Left: finite-elements-based discretizations (the present sketch illustrates the possibility, implemented in the open-source software $C A L I F^{3} S[10]$ developed at IRSN, of mixing simplicial (Crouzeix-Raviart) and quadrangular (Rannacher-Turek) cells) - Right: MAC discretization, dual cell for the y-component of the velocity.

When $K \in \mathcal{M}$ is a simplex, a rectangle or a cuboid, for $\sigma \in \mathcal{E}(K)$, we define $D_{K, \sigma}$ as the cone with basis $\sigma$ and with vertex the mass center of $K$ (see Figure 2.1). We thus obtain a partition of $K$ in $m$ sub-volumes, where $m$ is the number of faces of the mesh, each sub-volume having the same measure $\left|D_{K, \sigma}\right|=|K| / m$. We extend this definition to general quadrangles and hexahedra, by supposing that we have built a partition still of equal-volume sub-cells, and with the same connectivities. As shown in Figure 2.1, these sub-volumes may be of any shape; they do not need to be actually constructed when implementing the scheme. Note that this is of course always possible, but that such a volume $D_{K, \sigma}$ may be no longer a cone; indeed, if $K$ is far from a parallelogram, it may not be possible to build a cone having $\sigma$ as basis, the opposite vertex lying in $K$ and a volume equal to $|K| / m$. The volume $D_{K, \sigma}$ is referred to as the half-diamond cell associated to $K$ and $\sigma$. For $\sigma \in \mathcal{E}_{\text {int }}, \sigma=K \mid L$, we now define the diamond cell $D_{\sigma}$ associated to $\sigma$ by $D_{\sigma}=D_{K, \sigma} \cup D_{L, \sigma}$; for an external face $\sigma \in \mathcal{E}_{\text {ext }} \cap \mathcal{E}(K), D_{\sigma}$ is just the same volume as $D_{K, \sigma}$.

- Structured meshes: the MAC scheme [24, 23] - The degrees of freedom for the $i^{\text {th }}$ component of the velocity, $1 \leq i \leq d$, are defined at the centre of the faces $\sigma \in \mathcal{E}^{(i)}$ where $\mathcal{E}^{(i)} \subset \mathcal{E}$ is the subset of the faces of $\mathcal{E}$ which are perpendicular to the $i^{\text {th }}$ unit vector of the canonical basis of $\mathbb{R}^{d}$; so the whole set of discrete velocity unknowns reads:

$$
\left\{u_{\sigma, i}, \sigma \in \mathcal{E}^{(i)}, 1 \leq i \leq d\right\}
$$

For each component, the MAC dual mesh only differs from the RT or CR dual mesh by the choice of the half-diamond cell, which, for $K \in \mathcal{M}$ and $\sigma \in \mathcal{E}(K)$, is now the rectangle or rectangular parallelepiped of basis $\sigma$ and of measure $\left|D_{K, \sigma}\right|=|K| / 2$.

For any type of mesh, we denote by $\left|D_{\sigma}\right|$ the measure of the dual cell $D_{\sigma}$, and by $\epsilon=D_{\sigma} \mid D_{\sigma^{\prime}}$ the face separating two diamond cells $D_{\sigma}$ and $D_{\sigma^{\prime}}$. The set of the faces of a dual cell $D_{\sigma}$ is denoted by $\tilde{\mathcal{E}}\left(D_{\sigma}\right)$.

Finally, we need to deal with the wall boundary condition, i.e. $\boldsymbol{u} \cdot \boldsymbol{n}=0$. Since the velocity unknowns lie on the boundary (and not inside the cells), these conditions are taken into account in the definition of the discrete spaces. To avoid technicalities in the expression of the schemes, we suppose throughout this paper that the boundary is a.e. normal to a coordinate axis, (even in the case of the RT or CR discretizations), which allows to simply set to zero the corresponding velocity unknowns; defining by $\mathcal{E}_{\text {ext }}^{(i)} \subset \mathcal{E}_{\text {ext }}$ the subset of the faces of $\mathcal{E}_{\text {ext }}$ which are perpendicular to the $i^{\text {th }}$ unit 
vector of the canonical basis of $\mathbb{R}^{d}$, we set:

$$
\text { for } i=1, \ldots, d, \forall \sigma \in \mathcal{E}_{\mathrm{ext}}^{(i)}, \quad u_{\sigma, i}=0 .
$$

Therefore, there are no degrees of freedom for the velocity on the boundary for the MAC scheme, and there are only $d-1$ degrees of freedom on each boundary face for the CR and RT discretizations, which depend on the orientation of the face. In order to be able to write a unique expression of the discrete equations for both MAC and $\mathrm{CR} / \mathrm{RT}$ approximations, we introduce, for $1 \leq i \leq d$, the set of faces $\mathcal{E}_{\mathcal{S}}^{(i)}$ associated to the degrees of freedom of the $i^{\text {th }}$ component of the velocity ( $\mathcal{S}$ stands for "scheme"):

$$
\mathcal{E}_{\mathcal{S}}^{(i)}=\mid \begin{aligned}
& \mathcal{E}^{(i)} \backslash \mathcal{E}_{\text {ext }}^{(i)} \text { for the MAC scheme, } \\
& \mathcal{E} \backslash \mathcal{E}_{\text {ext }}^{(i)} \text { for the CR or RT schemes. }
\end{aligned}
$$

For both schemes, we define $\tilde{\mathcal{E}}^{(i)}$, for $1 \leq i \leq d$, as the set of faces of the dual mesh associated to the $i^{\text {th }}$ component of the velocity. For the RT or CR discretizations, the sets $\tilde{\mathcal{E}}^{(i)}$ does not depend on the component (i.e. of $i$ ), up to the elimination of some unknowns (and so some dual cells and, finally, some external faces) to take the boundary conditions into account. For the MAC scheme, $\tilde{\mathcal{E}}^{(i)}$ depends on $i$; note that each face of $\tilde{\mathcal{E}}^{(i)}$ is perpendicular to a unit vector of the canonical basis of $\mathbb{R}^{d}$, but not necessarily to the $i^{\text {th }}$ one.

General domains can be addressed (of course, with the CR or RT discretizations) by redefining, through linear combinations, the degrees of freedom at the external faces, so as to introduce the normal velocity as a new degree of freedom.

3. The numerical scheme. We build in this section a scheme for the Euler equations (1.1). We recall that the conservative energy equation of the system is the total energy equation:

$$
\partial_{t}(\rho E)+\operatorname{div}(\rho E \boldsymbol{u})+\operatorname{div}(p \boldsymbol{u})=0 .
$$

Let us suppose that the solution is regular, and let $E_{k}$ be the kinetic energy, defined by $E_{k}=\frac{1}{2}|\boldsymbol{u}|^{2}$. Taking the inner product of (1.1b) by $\boldsymbol{u}$ yields, after formal compositions of partial derivatives and using the mass balance (1.1a):

$$
\partial_{t}\left(\rho E_{k}\right)+\operatorname{div}\left(\rho E_{k} \boldsymbol{u}\right)+\nabla p \cdot \boldsymbol{u}=0 .
$$

This relation is referred to as the kinetic energy balance. Substracting this relation from the total energy balance (3.1), we obtain the internal energy balance equation:

$$
\partial_{t}(\rho e)+\operatorname{div}(\rho e \boldsymbol{u})+p \operatorname{div} \boldsymbol{u}=0 .
$$

Since,

- thanks to the mass balance equation, the first two terms in the left-hand side of (3.3) may be recast as a transport operator: $\partial_{t}(\rho e)+\operatorname{div}(\rho e \boldsymbol{u})=\rho\left[\partial_{t} e+\boldsymbol{u} \cdot \boldsymbol{\nabla} e\right]$, - and, from the equation of state, the pressure vanishes when $e=0$,

this equation implies, if $e \geq 0$ at $t=0$ and with suitable boundary conditions, that $e$ remains non-negative at all times. Solving this equation instead of the total energy equation seems appealing to preserve the positivity of the internal energy by construction of the scheme. Furthermore it avoids to introduce a discrete approximation for the total energy which would not be straightforward since the internal energy and the kinetic energy are not discretized on the same grid. We thus choose here to design a scheme solving the internal energy balance. However, the internal energy being a non-conservative variable, a raw discretization of (3.3) can lead to non-consistent solutions (wrong shock predictions for example). We overcome this difficulty by adding as in [27] a corrective term in the discrete internal energy balance equation; this point is discussed in Section 3.2 below. 
The presentation of the scheme is organized as follows. We first give the general form of the scheme (Section 3.1). Then we detail the construction of the corrective terms in the energy balance (Section 3.2). The next section (Section 3.3) is devoted to the stability analysis of the scheme; we prove that, under a CFL condition, the convex set of admissible states is preserved (so, in other words, $\rho>0, e>0$ and $p>0$ ) and show that the velocity and pressure are kept constant at the contact discontinuity. These results are obtained thanks to some abstract assumptions on the approximation of the density and internal energy at the face, in the discretization of the mass and internal energy convection term, respectively. We build in Section 3.4 a MUSCL type limiting procedure which allows to satisfy these assumptions, so that the density and energy convection operator is fully specified. Finally, Section 3.5 is devoted to the design of the artificial viscosity used to stabilize the scheme.

3.1. General form of the scheme. Let $\left(t_{n}\right)_{0 \leq n \leq N}$, with $0=t_{0}<t_{1}<\ldots<$ $t_{N}=T$, define a partition of the time interval $(0, T)$, which we suppose uniform for the sake of simplicity, and let $\delta t=t_{n+1}-t_{n}$ for $0 \leq n \leq N-1$ be the (constant) time step. We consider a fractional step scheme, which involves only explicit steps and reads in its fully discrete form, for $0 \leq n \leq N-1$ :

$$
\begin{aligned}
& \forall K \in \mathcal{M}, \frac{|K|}{\delta t}\left(\rho_{K}^{n+1}-\rho_{K}^{n}\right)+\sum_{\sigma \in \mathcal{E}(K)} F_{K, \sigma}^{n}=0, \\
& \forall K \in \mathcal{M}, \frac{|K|}{\delta t}\left(\rho_{K}^{n+1} e_{K}^{n+1}-\rho_{K}^{n} e_{K}^{n}\right)+\sum_{\sigma \in \mathcal{E}(K)} F_{K, \sigma}^{n} e_{\sigma}^{n}+|K| p_{K}^{n}(\operatorname{div} \boldsymbol{u})_{K}^{n}=S_{K}^{n}, \\
& \forall K \in \mathcal{M}, p_{K}^{n+1}=(\gamma-1) \rho_{K}^{n+1} e_{K}^{n+1},
\end{aligned}
$$

For $1 \leq i \leq d, \forall \sigma \in \mathcal{E}_{\mathcal{S}}^{(i)}$

$$
\begin{aligned}
\frac{\left|D_{\sigma}\right|}{\delta t}\left(\rho_{D_{\sigma}}^{n+1} u_{\sigma, i}^{n+1}-\rho_{D_{\sigma}}^{n} u_{\sigma, i}^{n}\right)+\sum_{\epsilon \in \tilde{\mathcal{E}}\left(D_{\sigma}\right)} F_{\sigma, \epsilon}^{n} u_{\epsilon, i}^{n} \\
+\sum_{\epsilon \in \tilde{\mathcal{E}}\left(D_{\sigma}\right)} \nu_{\epsilon}^{n+1}\left(u_{\sigma, i}^{n}-u_{\sigma^{\prime}, i}^{n}\right)+\left|D_{\sigma}\right|(\nabla p)_{\sigma, i}^{n+1}=0
\end{aligned}
$$

where the terms introduced for each discrete equation are defined hereafter.

Discrete mass balance. Equation (3.4a) is obtained by the discretization of the mass balance equation (1.1a) over the primal mesh, and $F_{K, \sigma}^{n}$ stands for the mass flux across $\sigma$ outward to the cell $K$, which, because of the wall condition, vanishes on external faces and is given on the internal faces by:

$$
\forall \sigma=K\left|L \in \mathcal{E}_{\text {int }}, \quad F_{K, \sigma}^{n}=\right| \sigma \mid \rho_{\sigma}^{n} u_{K, \sigma}^{n},
$$

where $u_{K, \sigma}^{n}$ is an approximation of the normal velocity to the face $\sigma$ to the cell $K$. This latter quantity is defined by:

$$
u_{K, \sigma}^{n}=\mid \begin{aligned}
& u_{\sigma, i}^{n} \boldsymbol{e}^{(i)} \cdot \boldsymbol{n}_{K, \sigma} \text { for } \sigma \in \mathcal{E}^{(i)} \text { in the MAC case, } \\
& \boldsymbol{u}_{\sigma}^{n} \cdot \boldsymbol{n}_{K, \sigma} \text { in the CR and RT cases, }
\end{aligned}
$$

where $\boldsymbol{e}^{(i)}$ denotes the $i^{\text {th }}$ vector of the orthonormal basis of $\mathbb{R}^{d}$. The density at the face $\sigma=K \mid L$ is approximated by a MUSCL type technique, detailed in Section 3.4. We only state here the algebraic condition which we require for this reconstruction, which is that for any $K \in \mathcal{M}$ and for any $\sigma \in \mathcal{E}(K) \cap \mathcal{E}_{\text {int }}$, there exists $\alpha_{\sigma}^{K} \in[0,1]$ and $M_{\sigma}^{K} \in \mathcal{M}$ such that:

$$
\rho_{\sigma}^{n}-\rho_{K}^{n}=\mid \begin{array}{ll}
\alpha_{\sigma}^{K}\left(\rho_{K}^{n}-\rho_{M_{\sigma}^{K}}\right) & \text { if } u_{K, \sigma}^{n} \geq 0 \\
\alpha_{\sigma}^{K}\left(\rho_{M_{\sigma}^{K}}-\rho_{K}^{n}\right) & \text { otherwise. }
\end{array}
$$


Note that this condition gives a mean to compute $\rho_{\sigma}^{n}$ in such a way that the scheme remains stable; it is, of course, satisfied by the upwind scheme, for which $\alpha_{\sigma}^{K}=$ 1 , and, for an edge $\sigma=K \mid L, M_{\sigma}^{K}$ is the upwind cell to $\sigma$, that is $M_{\sigma}^{K}=K$ if $u_{K, \sigma}^{n} \geq 0$ and $M_{\sigma}^{K}=L$ otherwise. It can be shown that in the one dimensional case, this condition is a consequence of the slope limitation procedure implemented for instance in the "minmod" and the "double minmod" schemes as defined in e.g. [42]. However, extending the technique of slope limitation to unstructured meshes in the multidimensional case is known to be intricate, while Condition (3.7) may be ensured by a simple limiting algorithm defining an admissible interval for the interface values, as in [34, section 3.2.2], see section 3.4 below.

Discrete momentum balance. The discrete momentum balance $(3.4 \mathrm{~d})$, is obtained by discretizing the momentum balance equation (1.1b) on the dual cells associated to the faces of the mesh. Up to the addition of the viscosity term $\sum_{\epsilon \in \tilde{\mathcal{E}}\left(D_{\sigma}\right)} \nu_{\epsilon}^{n+1}\left(u_{\sigma, i}^{n}-\right.$ $u_{\sigma^{\prime}, i}^{n}$ ) with $\nu_{\epsilon}^{n+1} \geq 0$, this equation is the same as in [27], and we refer to this work for details. The first task is to define the values $\rho_{D_{\sigma}}^{n+1}$ and $\rho_{D_{\sigma}}^{n}$, which approximate the density over the dual cell $D_{\sigma}$ at time $t^{n+1}$ and $t^{n}$ respectively, and the discrete mass flux through the dual face $\epsilon$ outward to the domain $D_{\sigma}$, denoted by $F_{\sigma, \epsilon}^{n}$; the guideline for their construction is that a finite volume discretization of the mass balance equation over the diamond cells, of the form

$$
\forall \sigma \in \mathcal{E}, \quad \frac{\left|D_{\sigma}\right|}{\delta t}\left(\rho_{D_{\sigma}}^{n+1}-\rho_{D_{\sigma}}^{n}\right)+\sum_{\epsilon \in \tilde{\mathcal{E}}\left(D_{\sigma}\right)} F_{\sigma, \epsilon}^{n}=0
$$

must hold in order to be able to derive a discrete kinetic energy balance (see Section 3.2 below). The density on the dual cells is given by the following weighted average:

$$
\begin{aligned}
& \text { for } \sigma=K \mid L \in \mathcal{E}_{\text {int }} \text {, for } k=n \text { and } k=n+1, \\
& \qquad\left|D_{\sigma}\right| \rho_{D_{\sigma}}^{k}=\left|D_{K, \sigma}\right| \rho_{K}^{k}+\left|D_{L, \sigma}\right| \rho_{L}^{k} .
\end{aligned}
$$

For the MAC scheme, the flux on a dual face is computed as follows [26]):

- if the dual face is located on two primal faces, then the dual flux is half the sum of the fluxes on the two primal faces;

- if the dual face is located in between two primal faces, the dual flux is again half of the sum of the fluxes on these two primal faces.

In the case of the CR and RT schemes, the flux through a dual face $\epsilon$ included in the primal cell $K$ is computed as a linear combination of the mass fluxes $\left(F_{K, \sigma}^{n}\right)_{\sigma \in \mathcal{E}(K)}$ defined by and the discrete mass balance (3.4a); note that the coefficients of this linear combination are constant: they do not depend on the cell itself, but only on the number of its faces. We refer to $[1,18]$ for a detailed construction of this approximation. Let us remark that a dual face lying on the boundary is then also a primal face, and the flux across this face is zero, because of the wall boundary condition. Therefore, the values $u_{\epsilon, i}^{n}$ are only needed at the internal dual faces, and we make the upwind choice for their discretization:

$$
\text { for } \epsilon=D_{\sigma}\left|D_{\sigma^{\prime}}, \quad u_{\epsilon, i}^{n}=\right| \begin{array}{ll}
u_{\sigma, i}^{n} & \text { if } F_{\sigma, \epsilon}^{n} \geq 0, \\
u_{\sigma^{\prime}, i}^{n} & \text { otherwise. }
\end{array}
$$

The last term $(\nabla p)_{\sigma, i}^{n+1}$ stands for the $i^{t h}$ component of the discrete pressure gradient at the face $\sigma$. The gradient operator is built as the transpose of the discrete operator for the divergence of the velocity, the discretization of which is based on the primal mesh. Let us denote the following natural approximation of the divergence of $\boldsymbol{u}^{n+1}$ over $K \in \mathcal{M}$ by $(\operatorname{div} \boldsymbol{u})_{K}^{n+1}$, with

$$
\text { for } K \in \mathcal{M}, \quad(\operatorname{div} \boldsymbol{u})_{K}^{n+1}=\frac{1}{|K|} \sum_{\sigma \in \mathcal{E}(K)}|\sigma| u_{K, \sigma}^{n+1} .
$$


The components of the pressure gradient are given by:

$$
\text { for } \sigma=K \mid L \in \mathcal{E}_{\mathrm{int}}, \quad(\boldsymbol{\nabla} p)_{\sigma, i}^{n+1}=\frac{|\sigma|}{\left|D_{\sigma}\right|}\left(p_{L}^{n+1}-p_{K}^{n+1}\right) \boldsymbol{n}_{K, \sigma} \cdot \boldsymbol{e}^{(i)},
$$

so that, thanks to the conservativity property $u_{K, \sigma}=-u_{L, \sigma}$ for $\sigma=K \mid L$, the following duality relation with respect to the $\mathrm{L}^{2}$ inner product:

$$
\sum_{K \in \mathcal{M}}|K| p_{K}^{n+1}(\operatorname{div} \boldsymbol{u})_{K}^{n+1}+\sum_{i=1}^{d} \sum_{\sigma \in \mathcal{E}_{\mathcal{S}}^{(i)}}\left|D_{\sigma}\right| u_{\sigma, i}^{n+1}(\nabla p)_{\sigma, i}^{n+1}=0
$$

Note that, because of the wall boundary conditions, the discrete gradient does not need to be defined at the external faces.

Discrete internal energy balance. Equation (3.4b) is an approximation of the internal energy balance over the primal cell $K$. For the discretization of the internal energy at the primal faces we use the same MUSCL technique as for the density to ensure the positivity of the convection operator, see Section 3.4; hence, for any $K \in \mathcal{M}$ and any $\sigma \in \mathcal{E}(K) \cap \mathcal{E}_{\text {int }}$, there exists $\alpha_{\sigma}^{K} \in[0,1]$ and $M_{\sigma}^{K} \in \mathcal{M}$ such that:

$$
e_{\sigma}^{n}-e_{K}^{n}=\mid \begin{aligned}
& \alpha_{\sigma}^{K}\left(e_{K}^{n}-e_{M_{\sigma}^{K}}^{n}\right), \text { if } F_{K, \sigma}^{n} \geq 0 \\
& \alpha_{\sigma}^{K}\left(e_{M_{\sigma}^{K}}^{n}-e_{K}^{n}\right), \text { otherwise }
\end{aligned}
$$

The discrete divergence of the velocity, $(\operatorname{div} \boldsymbol{u})_{K}^{n}$, is defined by $(3.11)$. The right-hand side, $S_{K}^{n}$, is derived using consistency arguments in the next section; at the first time step, it is simply set to zero:

$$
\forall K \in \mathcal{M}, \quad S_{K}^{0}=0 .
$$

Finally, the initial approximations for $\rho, e$ and $\boldsymbol{u}$ are given by the average of the initial conditions $\rho_{0}$ and $e_{0}$ on the primal cells and of $\boldsymbol{u}_{0}$ on the dual cells:

$$
\begin{aligned}
& \forall K \in \mathcal{M}, \quad \rho_{K}^{0}=\frac{1}{|K|} \int_{K} \rho_{0}(\boldsymbol{x}) \mathrm{d} \boldsymbol{x}, \quad \text { and } e_{K}^{0}=\frac{1}{|K|} \int_{K} e_{0}(\boldsymbol{x}) \mathrm{d} \boldsymbol{x}, \\
& \text { for } 1 \leq i \leq d, \forall \sigma \in \mathcal{E}_{\mathcal{S}}^{(i)}, \quad u_{\sigma, i}^{0}=\frac{1}{\left|D_{\sigma}\right|} \int_{D_{\sigma}}\left(\boldsymbol{u}_{0}(\boldsymbol{x})\right)_{i} \mathrm{~d} \boldsymbol{x} .
\end{aligned}
$$

These average values may be obtained by a quadrature formula if need be.

3.2. Discrete kinetic energy balance and corrective source terms. Equation (3.16) below is a discrete analogue of the kinetic energy balance equation (3.2), with some additional terms due to the artificial viscosity present in the scheme.

At the continuous level, the kinetic energy balance is obtained by taking the inner product of the momentum balance equation by the velocity and using twice the mass balance equation. At the discrete level, the computation is essentially the same for the convection term, provided that a momentum balance and a mass balance hold on the same cell, which is ensured by construction of the dual densities and mass fluxes (Relation (3.8)). For the artificial diffusion term implemented in the scheme, the algebraic manipulations performed at the discrete level are reminiscent of the continuous identity $-u_{i} \operatorname{div}\left(\mu \boldsymbol{\nabla} u_{i}\right)=-\operatorname{div}\left(\mu u_{i} \nabla u_{i}\right)+\mu\left|\nabla u_{i}\right|^{2}$. The conservative term is left at the left-hand side of the equation, while the dissipation term is considered as a residual.

LEMma 3.1 (Discrete kinetic energy balance). A solution to the system (3.4) 
satisfies the following equality, for $1 \leq i \leq d, \sigma \in \mathcal{E}_{\mathcal{S}}^{(i)}$ and $0 \leq n \leq N-1$ :

$$
\begin{aligned}
\frac{1}{2} \frac{\left|D_{\sigma}\right|}{\delta t}\left[\rho_{D_{\sigma}}^{n+1}\left(u_{\sigma, i}^{n+1}\right)^{2}-\rho_{D_{\sigma}}^{n}\left(u_{\sigma, i}^{n}\right)^{2}\right] & \\
+ & \frac{1}{2} \sum_{\epsilon=D_{\sigma} \mid D_{\sigma^{\prime}} \in \tilde{\mathcal{E}}\left(D_{\sigma}\right)} F_{\sigma, \epsilon}^{n} u_{\sigma, i}^{n} u_{\sigma^{\prime}, i}^{n}+\left|D_{\sigma}\right|(\nabla p)_{\sigma, i}^{n+1} u_{\sigma, i}^{n+1} \\
& +\frac{1}{2} \sum_{\epsilon=D_{\sigma} \mid D_{\sigma^{\prime}} \in \tilde{\mathcal{E}}\left(D_{\sigma}\right)} \mu_{\epsilon}^{n}\left(u_{\sigma, i}^{n}-u_{\sigma^{\prime}, i}^{n}\right)\left(u_{\sigma, i}^{n}+u_{\sigma^{\prime}, i}^{n}\right)=-R_{\sigma, i}^{n+1},
\end{aligned}
$$

with

$$
\begin{array}{r}
R_{\sigma, i}^{n+1}=\frac{1}{2} \frac{\left|D_{\sigma}\right|}{\delta t} \rho_{D_{\sigma}}^{n+1}\left(u_{\sigma, i}^{n+1}-u_{\sigma, i}^{n}\right)^{2}+\frac{1}{2} \sum_{\epsilon=D_{\sigma} \mid D_{\sigma^{\prime}} \in \tilde{\mathcal{E}}\left(D_{\sigma}\right)} \mu_{\epsilon}^{n}\left(u_{\sigma^{\prime}, i}^{n}-u_{\sigma, i}^{n}\right)^{2} \\
+\sum_{\epsilon=D_{\sigma} \mid D_{\sigma^{\prime}} \in \tilde{\mathcal{E}}\left(D_{\sigma}\right)}\left(\mu_{\epsilon}^{n}-\frac{F_{\sigma, \epsilon}}{2}\right)\left(u_{\sigma, i}^{n+1}-u_{\sigma, i}^{n}\right)\left(u_{\sigma, i}^{n}-u_{\sigma^{\prime}, i}^{n}\right),
\end{array}
$$

and where $\mu_{\epsilon}^{n}=\left|F_{\sigma, \epsilon}^{n}\right| / 2+\nu_{\epsilon}^{n+1}$.

Proof. We begin by rewriting the momentum balance equation (3.4d) so that the upwinding (3.10) now appears as a diffusion term. For $1 \leq i \leq d$ and $\sigma \in \mathcal{E}_{\mathcal{S}}^{(i)}$ and $0 \leq n \leq N-1$, we have:

$$
\begin{aligned}
\frac{\left|D_{\sigma}\right|}{\delta t}\left(\rho_{D_{\sigma}}^{n+1} u_{\sigma, i}^{n+1}-\rho_{D_{\sigma}}^{n} u_{\sigma, i}^{n}\right)+\sum_{\epsilon} F_{\sigma, \epsilon}^{n} & \frac{u_{\sigma, i}^{n}+u_{\sigma^{\prime}, i}^{n}}{2} \\
& +\sum_{\epsilon} \mu_{\epsilon}^{n}\left(u_{\sigma, i}^{n}-u_{\sigma^{\prime}, i}^{n}\right)+\left|D_{\sigma}\right|(\nabla p)_{\sigma, i}^{n+1}=0
\end{aligned}
$$

with $\mu_{\epsilon}^{n}=\left|F_{\sigma, \epsilon}^{n}\right| / 2+\nu_{\epsilon}^{n+1}$ and where, in this relation and throughout the proof, we denote by $\sum_{\epsilon}$ the sum over the internal faces of $D_{\sigma}$, the neighbouring diamond cell being $D_{\sigma^{\prime}}\left(\right.$ i.e. $\left.\epsilon=D_{\sigma} \mid D_{\sigma^{\prime}}\right)$. We multiply this relation by $u_{\sigma, i}^{n+1}$. The treatment of the first two terms (i.e. the time derivative and the convection term) is similar to the proof of [27, Lemma A.2], with differences induced by the fact that the discretization is centered; we give it here in extenso for the reader's convenience. To this purpose, we now remark that, thanks to the mass balance equation (3.8), for any families $\left(z_{\sigma}^{n}\right)$ and $\left(z_{\epsilon}^{n}\right), \sigma \in \mathcal{E}_{\mathcal{S}}^{(i)}, \epsilon \in \tilde{\mathcal{E}}_{\mathcal{S}}^{(i)}, 0 \leq n \leq N-1$, we have:

$$
\frac{\left|D_{\sigma}\right|}{\delta t}\left(\rho_{D_{\sigma}}^{n+1} z_{\sigma}^{n+1}-\rho_{D_{\sigma}}^{n} z_{\sigma}^{n}\right)+\sum_{\epsilon} F_{\sigma, \epsilon}^{n} z_{\epsilon}^{n}=\frac{\left|D_{\sigma}\right|}{\delta t} \rho_{D_{\sigma}}^{n+1}\left(z_{\sigma}^{n+1}-z_{\sigma}^{n}\right)+\sum_{\epsilon} F_{\sigma, \epsilon}^{n}\left(z_{\epsilon}^{n}-z_{\sigma}^{n}\right) .
$$

If $\left(z_{\sigma}^{n}\right)$ and $\left(z_{\epsilon}^{n}\right)$ are approximations of a continuous variable $z$, the left and right hand sides may be seen as a discretization of $\partial_{t}(\rho z)+\operatorname{div}(\rho z \boldsymbol{u})$ and $\rho \partial_{t} z+\rho \boldsymbol{u} \cdot \boldsymbol{\nabla} z$, respectively, so this simple computation is the discrete analogue of the passage from the conservative form to the non-conservative form of a balance equation. So, passing to the non-conservative form, we have:

$$
\begin{aligned}
\frac{\left|D_{\sigma}\right|}{\delta t}\left(\rho_{D_{\sigma}}^{n+1} u_{\sigma, i}^{n+1}-\rho_{D_{\sigma}}^{n} u_{\sigma, i}^{n}\right)+\sum_{\epsilon} & F_{\sigma, \epsilon}^{n} \frac{u_{\sigma, i}^{n}+u_{\sigma^{\prime}, i}^{n}}{2} \\
& =\frac{\left|D_{\sigma}\right|}{\delta t} \rho_{D_{\sigma}}^{n+1}\left(u_{\sigma, i}^{n+1}-u_{\sigma, i}^{n}\right)+\sum_{\epsilon} F_{\sigma, \epsilon}^{n} \frac{u_{\sigma^{\prime}, i}^{n}-u_{\sigma, i}^{n}}{2} .
\end{aligned}
$$

Multiplying this relation by $u_{\sigma, i}^{n+1}$, introducing $u_{\sigma, i}^{n}$ instead of $u_{\sigma, i}^{n+1}$ in factor of the convection term (with a residual term) and using twice the identity $2 a(a-b)=$ 
$a^{2}-b^{2}+(a-b)^{2}$, we get

$$
\begin{aligned}
T_{1}=\left[\frac{\left|D_{\sigma}\right|}{\delta t}\left(\rho_{D_{\sigma}}^{n+1} u_{\sigma, i}^{n+1}-\rho_{D_{\sigma}}^{n} u_{\sigma, i}^{n}\right)+\sum_{\epsilon} F_{\sigma, \epsilon}^{n} \frac{u_{\sigma, i}^{n}+u_{\sigma^{\prime}, i}^{n}}{2}\right] u_{\sigma, i}^{n+1} \\
=\frac{\left|D_{\sigma}\right|}{2 \delta t} \rho_{D_{\sigma}}^{n+1}\left(\left(u_{\sigma, i}^{n+1}\right)^{2}-\left(u_{\sigma, i}^{n}\right)^{2}\right)+\sum_{\epsilon} F_{\sigma, \epsilon}^{n} \frac{\left(u_{\sigma^{\prime}, i}^{n}\right)^{2}-\left(u_{\sigma, i}^{n}\right)^{2}}{4}+R_{1}+R_{2},
\end{aligned}
$$

with:

$$
\begin{aligned}
& R_{1}=\frac{\left|D_{\sigma}\right|}{2 \delta t} \rho_{D_{\sigma}}^{n+1}\left(u_{\sigma, i}^{n+1}-u_{\sigma, i}^{n}\right)^{2}+\left(u_{\sigma, i}^{n+1}-u_{\sigma, i}^{n}\right) \sum_{\epsilon} F_{\sigma, \epsilon}^{n} \frac{u_{\sigma, i}^{n}+u_{\sigma^{\prime}, i}^{n}}{2}, \\
& R_{2}=-\sum_{\epsilon} F_{\sigma, \epsilon}^{n} \frac{\left(u_{\sigma^{\prime}, i}^{n}-u_{\sigma, i}^{n}\right)^{2}}{4} .
\end{aligned}
$$

We now put $T_{1}$ under conservative form, using once again (3.18):

$$
T_{1}=\frac{\left|D_{\sigma}\right|}{2 \delta t}\left(\rho_{D_{\sigma}}^{n+1}\left(u_{\sigma, i}^{n+1}\right)^{2}-\rho_{D_{\sigma}}^{n}\left(u_{\sigma, i}^{n}\right)^{2}\right)+\sum_{\epsilon} F_{\sigma, \epsilon}^{n} \frac{\left(u_{\sigma^{\prime}, i}^{n}\right)^{2}+\left(u_{\sigma, i}^{n}\right)^{2}}{4}+R_{1}+R_{2} .
$$

Finally, we observe that the remainder term $R_{2}$ is conservative, and choose to add it in the convection term of $T_{1}$, which yields:

$$
T_{1}=\frac{\left|D_{\sigma}\right|}{2 \delta t}\left(\rho_{D_{\sigma}}^{n+1}\left(u_{\sigma, i}^{n+1}\right)^{2}-\rho_{D_{\sigma}}^{n}\left(u_{\sigma, i}^{n}\right)^{2}\right)+\sum_{\epsilon} F_{\sigma, \epsilon}^{n} \frac{u_{\sigma^{\prime}, i}^{n} u_{\sigma, i}^{n}}{2}+R_{1} .
$$

For the diffusion term, we just use the following elementary computation:

$$
\begin{aligned}
\left(u_{\sigma, i}^{n}-u_{\sigma^{\prime}, i}^{n}\right) u_{\sigma, i}^{n+1}=\left(u_{\sigma, i}^{n}-u_{\sigma^{\prime}, i}^{n}\right) & \left(u_{\sigma, i}^{n+1}-u_{\sigma, i}^{n}\right) \\
& +\frac{1}{2}\left(u_{\sigma, i}^{n}-u_{\sigma^{\prime}, i}^{n}\right)^{2}+\frac{1}{2}\left(u_{\sigma, i}^{n}-u_{\sigma^{\prime}, i}^{n}\right)\left(u_{\sigma, i}^{n}+u_{\sigma^{\prime}, i}^{n}\right) .
\end{aligned}
$$

The last term of this expression is conservative, and thus kept at the left-hand side of the discrete kinetic energy balance, while the other two terms are considered as residual terms, which yields the result.

The residual terms $R_{\sigma, i}^{n+1}$ may be seen as a numerical dissipation generated by the numerical diffusion. Because of the discontinuous solutions that exist in the case of the inviscid Euler equations, these terms do not tend to zero with the mesh and time steps, but subsist as measures borne by the shocks (see [20, Remark 4.1]), contrary to the numerical diffusion terms in conservative form, which do tend to zero. In order for the scheme to be consistent with the total energy balance, we thus need to compensate this dissipation in the internal energy balance by adding the corrective terms $S_{K}^{n}$ in (3.4b). Because of the staggered discretization, the kinetic energy balance is associated to the dual mesh while the internal energy balance is discretized on the primal mesh and we are not able to recover a local total energy balance since and a direct term-to-term compensation is not possible. Hence the correction terms $\left(S_{K}^{n+1}\right)$ are built by dispatching the terms $\left(R_{\sigma, i}^{n+1}\right)$ given by (3.17) on the neighbouring primal cells. For $K \in \mathcal{M}, S_{K}^{n+1}$ is computed as $S_{K}^{n+1}=\sum_{i=1}^{d} S_{K, i}^{n+1}$ with:

$$
S_{K, i}^{n+1}=\frac{1}{2} \rho_{K}^{n+1} \sum_{\sigma \in \mathcal{E}(K) \cap \mathcal{E}_{\mathcal{S}}^{(i)}} \frac{\left|D_{K, \sigma}\right|}{\delta t}\left(u_{\sigma, i}^{n+1}-u_{\sigma, i}^{n}\right)^{2}+\sum_{\epsilon \in \tilde{\mathcal{E}}_{\mathcal{S}}^{(i)}, \epsilon \cap \bar{K} \neq \emptyset} S_{K, \epsilon, i}^{n+1},
$$

where $S_{K, \epsilon, i}^{n+1}$ stands for the contribution of $\epsilon$ to $S_{K, i}^{n+1}$, which we now define.

Step 1 - To this purpose, our first task is, for a given dual face $\epsilon$, to gather the remainders (3.17) associated to the two neighbour dual cells. Let $\sigma_{\epsilon}^{u}$ and $\sigma_{\epsilon}^{d}$ be the two primal faces such that $\epsilon=D_{\sigma_{\epsilon}^{d}} \mid D_{\sigma_{\epsilon}^{u}}$ and $F_{\sigma_{\epsilon}^{d}, \epsilon}^{n} \leq 0$ (i.e. $D_{\sigma_{\epsilon}^{d}}$ is the dual cell 
located downstream $\epsilon$, see Figure 3.1). Then, we get for $\epsilon$ the following contribution from the upstream dual cell:

$$
\begin{aligned}
\left(R_{\epsilon, i}^{u}\right)^{n+1}=\frac{1}{2}\left(\frac{\left|F_{\sigma_{\epsilon}^{u}, \epsilon}^{n}\right|}{2}+\nu_{\epsilon}^{n+1}\right)\left(u_{\sigma_{\epsilon}^{u}, i}^{n}\right. & \left.-u_{\sigma_{\epsilon}^{d}, i}^{n}\right)^{2} \\
& +\nu_{\epsilon}^{n+1}\left(u_{\sigma_{\epsilon}^{u}, i}^{n+1}-u_{\sigma_{\epsilon}^{u}, i}^{n}\right)\left(u_{\sigma_{\epsilon}^{u}, i}^{n}-u_{\sigma_{\epsilon}^{d}, i}^{n}\right) .
\end{aligned}
$$

The contribution of the downstream dual cell reads:

$$
\begin{aligned}
\left(R_{\epsilon, i}^{d}\right)^{n+1}=\frac{1}{2}\left(\frac{\left|F_{\sigma_{\epsilon}^{d}, \epsilon}^{n}\right|}{2}+\nu_{\epsilon}^{n+1}\right)\left(u_{\sigma_{\epsilon}^{d}, i}^{n}-u_{\sigma_{\epsilon}^{u}, i}^{n}\right)^{2} & \\
& +\left(\left|F_{\sigma_{\epsilon}^{d}, \epsilon}^{n}\right|+\nu_{\epsilon}^{n+1}\right)\left(u_{\sigma_{\epsilon}^{d}, i}^{n+1}-u_{\sigma_{\epsilon}^{d}, i}^{n}\right)\left(u_{\sigma_{\epsilon}^{d}, i}^{n}-u_{\sigma_{\epsilon}^{u}, i}^{n}\right) .
\end{aligned}
$$

Gathering both terms, we obtain that $R_{\epsilon, i}^{n+1}=\left(R_{\epsilon, i}^{d}\right)^{n+1}+\left(R_{\epsilon, i}^{u}\right)^{n+1}$.

Step 2 - Let us now distribute $R_{\epsilon, i}^{n+1}$ in $S_{K, \epsilon, i}^{n+1}$. There are two different cases.

Case $\epsilon \subset K$. If $\epsilon$ is included in $K$, we just set $S_{K, \epsilon, i}^{n+1}=R_{\epsilon, i}^{n+1}$. This is the only situation to consider for the RT and CR discretizations. It is also the case for dual faces which are normal to $e^{(i)}$ for the MAC scheme.

Case $\epsilon$ partly included in $\partial K$. For the MAC scheme, some dual faces are partly included in $\partial K$, as depicted in Figure 3.1; let us first consider the case where $K$ is upstream to $\epsilon$ (or, in other words, the case where $\sigma_{\epsilon}^{u}$ is a face of $K$, see Figure 3.1). Let $L$ be the other upstream primal cell to $\epsilon$ (or, in other words, the cell such that $\left.\sigma_{\epsilon}^{u}=K \mid L\right)$. Then we set:

$$
\begin{aligned}
& S_{K, \epsilon, i}^{n+1}=\frac{|K|}{|K|+|L|}\left[\left(R_{\epsilon, i}^{u}\right)^{n+1}-\frac{\left|F_{\sigma_{\epsilon}^{u}, \epsilon}^{n}\right|}{4}\left(u_{\sigma_{\epsilon}^{u}, i}^{n}-u_{\sigma_{\epsilon}^{d}, i}^{n}\right)^{2}\right] \\
& =\frac{|K|}{|K|+|L|} \nu_{\epsilon}^{n+1}\left[\frac{1}{2}\left(u_{\sigma_{\epsilon}^{u}, i}^{n}-u_{\sigma_{\epsilon}^{d}, i}^{n}\right)^{2}+\left(u_{\sigma_{\epsilon}^{u}, i}^{n+1}-u_{\sigma_{\epsilon}^{u}, i}^{n}\right)\left(u_{\sigma_{\epsilon}^{u}, i}^{n}-u_{\sigma_{\epsilon}^{d}, i}^{n}\right)\right] .
\end{aligned}
$$

If $K$ is a downstream cell, we set:

$$
\begin{gathered}
S_{K, \epsilon, i}^{n+1}=\frac{|K|}{|K|+|L|}\left[\left(R_{\epsilon, i}^{d}\right)^{n+1}+\frac{\left|F_{\sigma_{\epsilon}^{u}, \epsilon}^{n}\right|}{4}\left(u_{\sigma_{\epsilon}^{d}, i}^{n}-u_{\sigma_{\epsilon}^{u}, i}^{n}\right)^{2}\right] \\
=\frac{|K|}{|K|+|L|}\left(\left|F_{\sigma_{\epsilon}^{d}, \epsilon}^{n}\right|+\nu_{\epsilon}^{n+1}\right)\left[\begin{array}{c}
\frac{1}{2}\left(u_{\sigma_{\epsilon}^{d}, i}^{n}-u_{\sigma_{\epsilon}^{u}, i}^{n}\right)^{2} \\
\left.+\left(u_{\sigma_{\epsilon}^{d}, i}^{n+1}-u_{\sigma_{\epsilon}^{d}, i}^{n}\right)\left(u_{\sigma_{\epsilon}^{d}, i}^{n}-u_{\sigma_{\epsilon}^{u}, i}^{n}\right)\right] .
\end{array}\right.
\end{gathered}
$$

The expression of the terms $\left(S_{K}^{n+1}\right)_{K \in \mathcal{M}}$ may be justified by showing that, with this choice, under some compactness assumptions, we may pass to the limit in the scheme to show that any possible limit of approximate solutions is indeed a weak solution to the Euler equations. We may already note here that:

$$
\sum_{K \in \mathcal{M}} S_{K}^{n+1}-\sum_{i=1}^{d} \sum_{\sigma \in \mathcal{E}_{\mathcal{S}}^{(i)}} R_{\sigma, i}^{n+1}=0
$$

and so, by summing the kinetic energy balance over the component and faces and the internal energy balance over the cells, we observe that the integral of the total energy over the domain $\Omega$ is conserved. As already mentioned, a local conservation is more difficult to obtain because of the staggered discretization: the internal energy is defined on the primal cells and the kinetic energy on the dual cells. 


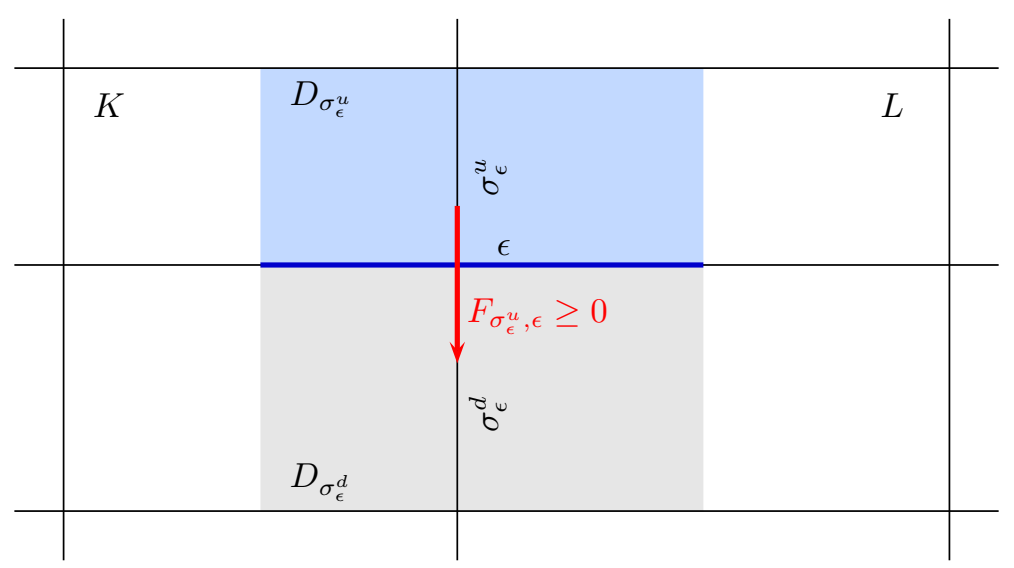

FIG. 3.1. Notations for the construction of the corrective term $S_{K, 1}$, in the MAC case, for a dual face lying on the primal cells boundaries. $\epsilon$ : considered dual edge. $D_{\sigma_{\epsilon}^{u}}$ : upstream dual cell. $D_{\sigma_{\epsilon}^{d}}$ : downstream dual cell. $K, L$ : upstream cells.

3.3. Stability results. The following positivity result is a consequence of the MUSCL interpolation of the density in (3.4a), as proved in [34, Theorem 3.2].

LEMMa 3.2 (Positivity of the density). Let $\rho^{0}$ be given by $(3.15)$. Then, since $\rho_{0}$ is assumed to be a positive function, $\rho^{0}>0$. Let us assume that the following $\mathrm{CFL}$ condition holds:

$$
\delta t \leq \frac{|K|}{\sum_{\sigma \in \mathcal{E}(K)}|\sigma|\left(1+\alpha_{\sigma}^{K}\right)\left(u_{K, \sigma}^{n}\right)^{+}}, \quad \forall K \in \mathcal{M}, \text { for } 0 \leq n \leq N-1
$$

where, for $a \in \mathbb{R}, a^{+} \geq 0$ is defined by $a^{+}=\max (a, 0)$ and $\alpha_{\sigma}^{K}$ is introduced in (3.7). Then the solution to the scheme (3.4) satisfies $\rho^{n}>0$, for $1 \leq n \leq N$.

The MUSCL interpolation of the internal energy at the face, together with the definition (3.19) of $\left(S_{K}^{n+1}\right)_{K \in \mathcal{M}}$ allow to prove that, under a CFL condition, the scheme also preserves the positivity of $e$.

LEMma 3.3 (Positivity of the internal energy). We assume that the CFL condition (3.25) holds. In addition, let the following additional CFL relations be satisfied for $0 \leq n \leq N-1$ :

$$
\begin{aligned}
& \delta t \leq \frac{|K| \rho_{K}^{n}}{(\gamma-1) \rho_{K}^{n} \sum_{\sigma \in \mathcal{E}(K)}|\sigma|\left(u_{K, \sigma}^{n}\right)^{+}+\sum_{\sigma \in \mathcal{E}(K)}\left(1+\alpha_{\sigma}^{K}\right)\left(F_{K, \sigma}^{n}\right)^{+}}, \quad \forall K \in \mathcal{M}, \\
& \delta t \leq \frac{\left|D_{K, \sigma}\right| \rho_{K}^{n}}{\sum_{\epsilon \in \tilde{\mathcal{E}}\left(D_{\sigma}\right), \epsilon \cap \bar{K} \neq \emptyset} \nu_{\epsilon}^{n+1}+\left|F_{\sigma, \epsilon}^{n}\right|}, \quad \forall K \in \mathcal{M}, \forall \sigma \in \mathcal{E}(K) .
\end{aligned}
$$

Then the solution to the scheme (3.4) satisfies $e^{n}>0$, for $1 \leq n \leq N$.

Proof. Let $n$ such that $0<n \leq N-1$ be given, and let us assume in a first step that $e_{K}^{n} \geq 0$ and $S_{K}^{n} \geq 0$ for all $K \in \mathcal{M}$. Because (3.25) is satisfied we have $\rho_{K}^{n} \geq 0$ and $\rho_{K}^{n+1} \geq 0$. The definition (3.14) of the internal energy at the face yields that, for $K \in \mathcal{M}$ and $\sigma \in \mathcal{E}(K)$, there exists $\alpha_{\sigma}^{K} \in[0,1]$ and $M_{\sigma}^{K} \in \mathcal{M}$ such that:

$$
F_{K, \sigma}^{n} e_{\sigma}^{n}=\left(F_{K, \sigma}^{n}\right)^{+}\left[\left(\alpha_{\sigma}^{K}+1\right) e_{K}^{n}-\alpha_{\sigma}^{K} e_{M_{\sigma}^{K}}^{n}\right]-\left(F_{K, \sigma}^{n}\right)^{-}\left[\left(1-\alpha_{\sigma}^{K}\right) e_{K}^{n}+\alpha_{\sigma}^{K} e_{M_{\sigma}^{K}}^{n}\right]
$$

where, for $a \in \mathbb{R}, a^{-}=-\min (a, 0)$. Let us use this expression in the internal energy 
balance (3.4b), expressing the pressure thanks to the equation of state (3.4c) to obtain:

$$
\begin{aligned}
& \frac{|K|}{\delta t} \rho_{K}^{n+1} e_{K}^{n+1}= {\left[\frac{|K|}{\delta t} \rho_{K}^{n}-\sum_{\sigma \in \mathcal{E}(K)}\left(1+\alpha_{\sigma}^{K}\right)\left(F_{K, \sigma}^{n}\right)^{+}-(\gamma-1) \rho_{K}^{n} \sum_{\sigma \in \mathcal{E}(K)}|\sigma|\left(u_{K, \sigma}^{n}\right)^{+}\right] e_{K}^{n} } \\
&+ \sum_{\sigma \in \mathcal{E}(K)} \alpha_{\sigma}^{K}\left|F_{K, \sigma}^{n}\right| e_{M_{\sigma}^{K}}^{n} \\
&+\sum_{\sigma \in \mathcal{E}(K)}\left(1-\alpha_{\sigma}^{K}\right)\left(F_{K, \sigma}^{n}\right)^{-} e_{K}^{n} \\
&+(\gamma-1) \rho_{K}^{n} e_{K}^{n} \sum_{\sigma \in \mathcal{E}(K)}|\sigma|\left(u_{K, \sigma}^{n}\right)^{-}+S_{K}^{n} .
\end{aligned}
$$

Then we get $e_{K}^{n+1}>0$ under the CFL condition (3.26a).

Let us now derive a condition for the non-negativity of the source term $S_{K}^{n}$. We begin by deriving a lower bound for the contributions associated to the diffusion (artificial diffusion or consequence of the upwinding) at the dual faces. Let $i \in \llbracket 1, d \rrbracket$ and $\epsilon=D_{\sigma} \mid D_{\sigma^{\prime}} \in \tilde{\mathcal{E}}_{\mathcal{S}}^{(i)}$ be such that $\epsilon \cap \bar{K} \neq \emptyset$. Let us first consider the case of an internal dual face, where this quantity $S_{K, \epsilon, i}^{n}$ is the sum of the two terms given by (3.20) and (3.21). Both $\sigma$ and $\sigma^{\prime}$ are thus faces of $K$. Supposing, without loss of generality, that $D_{\sigma}$ (resp. $D_{\sigma^{\prime}}$ ) is the upstream (resp. downstream) dual cell, we get:

$$
\begin{aligned}
S_{K, \epsilon, i}^{n}=\left(\frac{\left|F_{\sigma, \epsilon}^{n}\right|}{2}+\nu_{\epsilon}^{n+1}\right)\left(u_{\sigma, i}^{n}-u_{\sigma^{\prime}}^{n}\right)^{2} & +\nu_{\epsilon}^{n+1}\left(u_{\sigma, i}^{n+1}-u_{\sigma, i}^{n}\right)\left(u_{\sigma, i}^{n}-u_{\sigma^{\prime}, i}^{n}\right) \\
& +\left(\left|F_{\sigma, \epsilon}^{n}\right|+\nu_{\epsilon}^{n+1}\right)\left(u_{\sigma^{\prime}, i}^{n+1}-u_{\sigma^{\prime}, i}^{n}\right)\left(u_{\sigma^{\prime}, i}^{n}-u_{\sigma, i}^{n}\right) .
\end{aligned}
$$

By Young's inequality, we thus get:

$$
S_{K, \epsilon, i}^{n} \geq-\frac{\nu_{\epsilon}^{n+1}}{2}\left(u_{\sigma, i}^{n+1}-u_{\sigma, i}^{n}\right)^{2}-\frac{\left|F_{\sigma, \epsilon}^{n}\right|+\nu_{\epsilon}^{n+1}}{2}\left(u_{\sigma^{\prime}, i}^{n+1}-u_{\sigma^{\prime}, i}^{n}\right)^{2} .
$$

We now turn, for the MAC scheme only, to the case of dual faces which partly lie on the boundary of the cell $K$. Let $\sigma$ be a face of $K$ (so that $\sigma^{\prime}$ is not). The quantity $S_{K, \epsilon, i}^{n}$ is now given either by $(3.22)$ or $(3.23)$ and takes the form

$$
S_{K, \epsilon, i}^{n}=\xi\left[\frac{1}{2}\left(u_{\sigma, i}^{n}-u_{\sigma^{\prime}, i}^{n}\right)^{2}+\left(u_{\sigma, i}^{n+1}-u_{\sigma, i}^{n}\right)\left(u_{\sigma, i}^{n}-u_{\sigma^{\prime}, i}^{n}\right)\right]
$$

with $\xi \leq\left|F_{\sigma, \epsilon}^{n}\right|+\nu_{\epsilon}^{n+1}$. So, once again by Young's inequality:

$$
S_{K, \epsilon, i}^{n} \geq-\frac{\left|F_{\sigma, \epsilon}^{n}\right|+\nu_{\epsilon}^{n+1}}{2}\left(u_{\sigma, i}^{n+1}-u_{\sigma, i}^{n}\right)^{2}
$$

Reordering the terms in (3.19) then yields:

$$
S_{K, i}^{n} \geq \sum_{\sigma \in \mathcal{E}(K) \cap \mathcal{E}_{\mathcal{S}}^{(i)}}\left[\frac{1}{2} \rho_{K}^{n+1} \frac{\left|D_{K, \sigma}\right|}{\delta t}-\frac{1}{2} \sum_{\epsilon \in \tilde{\mathcal{E}}\left(D_{\sigma}\right), \epsilon \cap \bar{K} \neq \emptyset} \nu_{\epsilon}^{n+1}+\left|F_{\sigma, \epsilon}\right|^{n}\right]\left(u_{\sigma, i}^{n+1}-u_{\sigma, i}^{n}\right)^{2}
$$

The positivity of $S_{K, i}^{n}$ is then ensured, provided that:

$$
\delta t \leq \min _{\sigma \in \mathcal{E}(K)} \frac{\left|D_{K, \sigma}\right| \rho_{K}^{n+1}}{\sum_{\epsilon \in \tilde{\mathcal{E}}\left(D_{\sigma}\right), \epsilon \cap \bar{K} \neq \emptyset} \nu_{\epsilon}^{n+1}+\left|F_{\sigma, \epsilon}^{n}\right|}
$$

This concludes the proof.

The upwind version of the scheme studied in [27] preserves the contact discontinuities if the pressure is a function of the product $\rho e$, which is the case of the perfect gas EOS (1.1d) considered here; indeed, if the pressure and velocity are constant through a contact discontinuity at time $t_{n}$, then they remain so at time $t_{n+1}$. We show in Proposition 3.4 below that under a condition which correlates the MUSCL reconstructions of the face values $e_{\sigma}$ and $\rho_{\sigma}$, the scheme (1.1a)-(1.1b) also preserves 
1D contact discontinuities. A MUSCL-like scheme which ensures this condition is presented in Section 3.4 below; note that the proposition below is proved for a perfect gas EOS; however it holds for any EOS such that $p$ is a function of the product $\rho e$.

Proposition 3.4 (Preservation of the contact discontinuities). Let us suppose that $u_{0}=u$ and $p_{0}=p, u$ and $p$ constant. Additionally assume that

$$
\begin{aligned}
\forall n \in \llbracket 1, N \rrbracket, \forall \sigma \in \mathcal{E}_{\text {int }}, \sigma=K \mid L, \exists \kappa_{\sigma}^{n} \in[0,1] ; & \rho_{\sigma}^{n} e_{\sigma}^{n} \\
& =\kappa_{\sigma}^{n} \rho_{K}^{n} e_{K}^{n}+\left(1-\kappa_{\sigma}^{n}\right) \rho_{L}^{n} e_{L}^{n},
\end{aligned}
$$

then $\forall n \in \llbracket 1, N \rrbracket$ and $\forall K \in \mathcal{M}, u_{K}^{n}=u$ and $p_{K}^{n}=p$.

Proof. Without loss of generality, we restrict ourselves to the one-dimensional case. A cell $K \in \mathcal{M}$ is then denoted $K=\left[\sigma^{\prime}, \sigma\right]$, where $\sigma^{\prime}$ and $\sigma$ are the two interfaces of $K$. Assume that the proposition is true for all $k \in \llbracket 0, n \rrbracket$ and for all $K=\left[\sigma^{\prime}, \sigma\right] \in \mathcal{M}$. It is easy to see that $S_{K}^{n}=0$ and $(\operatorname{div} u)_{K}^{n}=0$. The internal energy equation $(3.4 \mathrm{~b})$ for $K=\left[\sigma^{\prime}, \sigma\right]$ then reads:

$$
\frac{|K|}{\delta t}\left(\rho_{K}^{n+1} e_{K}^{n+1}-\rho_{K}^{n} e_{K}^{n}\right)+u\left(\rho_{\sigma}^{n} e_{\sigma}^{n}-\rho_{\sigma^{\prime}}^{n} e_{\sigma^{\prime}}^{n}\right)=0 .
$$

From the $\operatorname{EOS}(3.4 \mathrm{c})$, we get that $\rho_{K}^{n} e_{K}^{n}=\frac{p}{\gamma-1}, \quad \forall K \in \mathcal{M}$, and so

$$
\rho_{\sigma}^{n} e_{\sigma}^{n}=\kappa_{\sigma}^{n} \rho_{K}^{n} e_{K}^{n}+\left(1-\kappa_{\sigma}^{n}\right) \rho_{L}^{n} e_{L}^{n}=\frac{p}{\gamma-1}, \quad \forall \sigma \in \mathcal{E}_{\text {int }}, \sigma=K \mid L .
$$

Thus: $\rho_{K}^{n+1} e_{K}^{n+1}=\rho_{K}^{n} e_{K}^{n}$ and $p_{K}^{n+1}=p, \quad \forall K \in \mathcal{M}$, and $\nabla p_{\sigma}^{n+1}=0 \quad \forall \sigma \in \mathcal{E}_{\text {int }}, \sigma=$ $K \mid L$. Denoting by $F_{K}^{n}$ and $F_{L}^{n}$ the numerical fluxes $F_{\sigma, \epsilon}^{n}$ on the dual interfaces $\epsilon$ included in $K$ and $L$ respectively, and noting that $u_{\epsilon}^{n}=u$ for both interfaces, the momentum equation $(3.4 \mathrm{~d})$ then reads:

$$
\frac{\left|D_{\sigma}\right|}{\delta t}\left(\rho_{D_{\sigma}}^{n+1} u_{D_{\sigma}}^{n+1}-\rho_{D_{\sigma}}^{n} u_{D_{\sigma}}^{n}\right)+\left(F_{K}^{n}-F_{L}^{n}\right) u=0 .
$$

Together with the discrete dual mass balance (3.8) which reads

$$
\frac{\left|D_{\sigma}\right|}{\delta t}\left(\rho_{D_{\sigma}}^{n+1}-\rho_{D_{\sigma}}^{n}\right)+\left(F_{K}^{n}-F_{L}^{n}\right)=0
$$

we obtain that

$$
\frac{\left|D_{\sigma}\right|}{\delta t} \rho_{D_{\sigma}}^{n+1}\left(u_{\sigma}^{n+1}-u\right)=0
$$

and therefore $u_{\sigma}^{n+1}=u \forall \sigma \in \mathcal{E}_{\text {int }}$, which concludes the proof of the proposition.

3.4. A MUSCL-like interpolation. As already mentioned when introducing the general form of the scheme (3.4), the upwinding process is performed equation-perequation, on the basis of the material velocity only; a MUSCL-like strategy is applied only for the density and internal energy balance equations. It is not applied on the momentum balance for the following reasons: first of all, as already mentioned, most of the numerical diffusion occurs at the contact discontinuities, where the velocity is constant. To increase the accuracy or the scheme, it is therefore natural to implement the MUSCL procedure on the density and internal energy which are discontinuous at the contact discontinuities. Moreover, the kinetic energy remainder contains a negative term from the time discretization and a positive term from the numerical space diffusion; if the latter one is reduced by a MUSCL-like procedure, then the sign of the remainder is no longer controlled. Also, the scheme is only stabilized by an artificial diffusion in the momentum equation, and this diffusion must be important enough to kill the oscillations. Experience shows that this procedure gives better results than adding diffusion everywhere. Finally, this additional diffusion in the momentum balance helps to recover an entropy inequality, as we recently showed in 
[17]. The objective of this section is to detail this algorithm, thus, precisely speaking, the approximation of the density and internal energy at the primal face in equations (3.4a) and (3.4b) respectively.

As a consequence of this equation-per-equation process, the problem that we face is close to the program realized in [34], namely to build an approximation for a convection operator (satisfying a maximum principle) which is formally second order in space when the solution is regular, and preserves the range of variation of the unknowns even in case of shocks, by an adequate flux limiting procedure. The algorithm presented here is thus an extension of the scheme developped in [34]; in particular, contrary to most MUSCL reconstructions which use slope estimation and limiting, see e.g. [3, 39] for reviews and $[31,5,8,9]$ for recent works, the limiting technique is here directly derived from stability conditions which are purely algebraic, in the sense that they do not require any geometric computation, and thus work with arbitrary meshes.

Compared to [34], the algorithm is however complicated by the requirement that the scheme should preserve pressure-constant zones, to avoid to destabilize the computation of contact discontinuities, or more precisely, of the one-dimensional contact discontinuity, across which the velocity is constant, the more difficult problem posed by slip interfaces in $2 \mathrm{D}$ or $3 \mathrm{D}$ being out of the scope of this study. In fine, this is realized by imposing to the face pressure (i.e. the pressure obtained by applying the equation of state to the face density and internal energy) to be a convex interpolation of the pressure in the two neighbouring cells. This condition leads to a limiting procedure which takes into account both mass and internal energy equations, so that we somehow loose here our equation decoupling strategy.

As often in MUSCL techniques, the algorithm consists in two steps: first compute a tentative second-order approximation (here for the density only) and then apply a limiting procedure. We describe these two steps successively in the following. For the sake of clarity, we omit in this section all the superscripts relative to the time step number.

Computation of a tentative value for the density - For an edge $\sigma \in \mathcal{E}_{\text {int }}$ and $K \in \mathcal{M}$, let us call $\boldsymbol{x}_{\sigma}$ and $\boldsymbol{x}_{K}$ the mass center of $\sigma$ and $K$ respectively. Let $\sigma \in \mathcal{E}_{\text {int }}$ be a given internal face. We suppose that we have computed a set of real coefficients $\left(\zeta_{\sigma}^{L}\right)$ such that:

$$
\boldsymbol{x}_{\sigma}=\sum_{L \in \mathcal{M}} \zeta_{\sigma}^{L} \boldsymbol{x}_{L}, \quad \sum_{L \in \mathcal{M}} \zeta_{\sigma}^{L}=1 .
$$

Then, for a known $\rho_{\mathcal{M}}=\left(\rho_{K}\right)_{K \in \mathcal{M}}$, we define the interpolation of the density at the face $\tilde{\rho}_{\sigma}$ by:

$$
\forall \sigma \in \mathcal{E}_{\text {int }}, \quad \tilde{\rho}_{\sigma}=\sum_{L \in \mathcal{M}} \zeta_{\sigma}^{L} \rho_{L}
$$

In practice, the cells $L \in \mathcal{M}$ used in Relation (3.28) are chosen as close as possible to $\sigma$, and a convex interpolation (i.e. positive real numbers $\left(\zeta_{\sigma}^{L}\right)$ ) is preferred each time it is possible. For structured discretization, the value at the internal face $\sigma=K \mid L$ is obtained as a weighted average of $\rho_{K}$ and $\rho_{L}$. For a detailed description of the implemented interpolation algorithm in the general case, the reader is referred to [34].

Limiting procedure - Let $\sigma \in \mathcal{E}_{\text {int }}, \sigma=K \mid L$, and let us suppose that the flow goes from $K$ to $L$, i.e. $F_{K, \sigma} \geq 0$. We now recall the conditions which were used to prove that the density and the internal energy remain positive, gathering the condition used for the cell $K$ and the condition used for $L$. For the density, we get that there exists $\alpha_{\sigma}^{\rho} \in[0,1], \beta_{\sigma}^{\rho} \in[0,1]$ and $M_{\sigma}^{\rho} \in \mathcal{M}$ such that

$$
\mid \begin{aligned}
& \rho_{\sigma}-\rho_{K}=\alpha_{\sigma}^{\rho}\left(\rho_{K}-\rho_{M_{\sigma}^{\rho}}\right), \\
& \rho_{\sigma}-\rho_{L}=\beta_{\sigma}^{\rho}\left(\rho_{K}-\rho_{L}\right) .
\end{aligned}
$$


Similarly, we have for the internal energy that there exists $\alpha_{\sigma}^{e} \in[0,1], \beta_{\sigma}^{e} \in[0,1]$ and $M_{\sigma}^{e} \in \mathcal{M}$ such that

$$
\mid \begin{aligned}
& e_{\sigma}-e_{K}=\alpha_{\sigma}^{e}\left(e_{K}-e_{M_{\sigma}^{\rho}}\right), \\
& e_{\sigma}-e_{L}=\beta_{\sigma}^{e}\left(e_{K}-e_{L}\right) .
\end{aligned}
$$

For the sake of simplicity, we suppose that the "upstream cells" $M_{\sigma}^{\rho}$ and $M_{\sigma}^{e}$ are the same and, from now on, we denote this cell by $M_{\sigma}$. We have shown in [34] that Equation (3.30) (respectively Equation (3.31)) defines an admissible interval for $\rho_{\sigma}$ (resp. $\left.e_{\sigma}\right)$, and that a limiting procedure may be obtained by just projecting the tentative value for the density $\tilde{\rho}_{\sigma}$ (resp. internal energy $\tilde{e}_{\sigma}$ ) at the face on this interval. Here, the situation is more complicated, since we also need to comply with the condition required for the scheme to keep the pressure constant at contact discontinuities, which states that the product $\rho_{\sigma} e_{\sigma}$ must be equal to $\rho_{K} e_{K}$ and $\rho_{L} e_{L}$ when these quantities are the same (recall that we use here the fact that the equation of state is such that the pressure only depends on the product $\rho e$ ). In fact, we require here the more restrictive assumption that $\rho_{\sigma} e_{\sigma}$ is a convex combination of $\rho_{K} e_{K}$ and $\rho_{L} e_{L}$, i.e. that there exists $\kappa_{\sigma} \in[0,1]$ so that:

$$
\rho_{\sigma} e_{\sigma}=\kappa_{\sigma} \rho_{K} e_{K}+\left(1-\kappa_{\sigma}\right) \rho_{L} e_{L}
$$

Our aim is now to find an admissible interval for $\rho_{\sigma}$ and $e_{\sigma}$ such that (3.30), (3.31) and (3.32) hold.

Let us first deal with(3.30). Combining both relations, we obtain that $\alpha_{\sigma}^{\rho}$ and $\beta_{\sigma}^{\rho}$ satisfy:

$$
\beta_{\sigma}^{\rho}=1-\frac{\alpha_{\sigma}^{\rho}}{r_{\sigma}^{\rho}}, \quad \text { with } r_{\sigma}^{\rho}=\frac{\rho_{L}-\rho_{K}}{\rho_{K}-\rho_{M_{\sigma}}}
$$

From this relation, it appears that (3.30) is satisfied (or, in other words, $\alpha_{\sigma}^{\rho} \in[0,1]$ and $\left.\beta_{\sigma}^{\rho} \in[0,1]\right)$ provided that $\alpha_{\sigma}^{\rho}$ satisfies:

$$
0 \leq \alpha_{\sigma}^{\rho} \leq\left(\min \left(1, r_{\sigma}^{\rho}\right)\right)^{+}
$$

This observation suggests the following strategy: thanks to the link between the value of $\rho_{\sigma}$ and $e_{\sigma}$ induced by Equation (3.32), try to express the coefficients $\alpha_{\sigma}^{e}$ and $\beta_{\sigma}^{e}$ as a fonction of $\alpha_{\sigma}^{\rho}$, and then express the limiting procedures produced by $(3.31)$ as limiting procedures for $\alpha_{\sigma}^{\rho}$. To this purpose, we remark that the second relation of (3.30) reads $\rho_{\sigma}=\beta_{\sigma}^{\rho} \rho_{K}+\left(1-\beta_{\sigma}^{\rho}\right) \rho_{L}$, and arbitrarily suppose that the product $\rho_{\sigma} e_{\sigma}$ is given by the same interpolation between neighbouring cells values:

$$
\rho_{\sigma} e_{\sigma}=\beta_{\sigma}^{\rho} \rho_{K} e_{K}+\left(1-\beta_{\sigma}^{\rho}\right) \rho_{L} e_{L}
$$

i.e. we take $\kappa=\beta_{\sigma}^{\rho}$ in (3.32). Note that many other choices would be possible, as, for instance, $\kappa=\beta_{\sigma}^{e}$. Dividing by $\rho_{\sigma}$ yields:

$$
e_{\sigma}=\frac{\beta_{\sigma}^{\rho} \rho_{K}}{\rho_{\sigma}} e_{K}+\frac{\left(1-\beta_{\sigma}^{\rho}\right) \rho_{L}}{\rho_{\sigma}} e_{L} .
$$

Since the right hand side may be seen as a convex interpolation between $e_{K}$ and $e_{L}$, we get that

$$
\beta_{\sigma}^{e}=\frac{\rho_{K}}{\rho_{\sigma}} \beta_{\sigma}^{\rho}
$$

and also that $\beta_{\sigma}^{e} \in[0,1]$, which may also be inferred directly from the fact that $\rho_{\sigma}=\beta_{\sigma}^{\rho} \rho_{K}+\left(1-\beta_{\sigma}^{\rho}\right) \rho_{L} \geq \beta_{\sigma}^{\rho} \rho_{K}$. From (3.31), we derive the following relation, which is the analogue of $(3.33)$ :

$$
\beta_{\sigma}^{e}=1-\frac{\alpha_{\sigma}^{e}}{r_{\sigma}^{e}}, \quad \text { with } r_{\sigma}^{e}=\frac{e_{L}-e_{K}}{e_{K}-e_{M_{\sigma}}} .
$$


Therefore, $\alpha_{\sigma}^{e}=\left(1-\beta_{\sigma}^{e}\right) r_{\sigma}^{e}$, and substituting $\beta_{\sigma}^{e}$ by its expression (3.34) and then expressing $\beta_{\sigma}^{\rho}$ as a function of $\alpha_{\sigma}^{\rho}$ thanks to (3.33) yields, after some agebraic manipulations:

$$
\alpha_{\sigma}^{e}=\frac{\rho_{L}}{\rho_{\sigma}} \frac{r_{\sigma}^{e}}{r_{\sigma}^{\rho}} \alpha_{\sigma}^{\rho}
$$

From this expression, and since we have already proven the existence of $\beta_{\sigma}^{e} \in[0,1]$, we get that there exists $\alpha_{\sigma}^{e} \in[0,1]$ such that (3.31), together with (3.30), are satisfied if $\alpha_{\sigma}^{\rho}$ is such that:

$$
0 \leq \alpha_{\sigma}^{\rho} \leq\left(\min \left(1, r_{\sigma}^{\rho}, \frac{\rho_{\sigma}}{\rho_{L}} \frac{r_{\sigma}^{\rho}}{r_{\sigma}^{e}}\right)\right)^{+} .
$$

This relation still does not provide an interval for $\alpha_{\sigma}^{\rho}$, since it involves $\rho_{\sigma}$ which expression itself involves $\alpha_{\sigma}^{\rho}$. But now, we just needto replace $\rho_{\sigma}$ by an explicit lower bound. As we already remarked, $\alpha_{\sigma}^{\rho}=0$ is always an admissible value, and so $\rho_{K}$ is also an admissible value for $\rho_{\sigma}$. Thus $\rho_{\sigma}$ will be obtained by a projection of the tentative value $\tilde{\rho}_{\sigma}$ on an interval containing $\rho_{K}$, which ensures that $\rho_{\sigma} \geq \min \left(\rho_{K}, \tilde{\rho}_{\sigma}\right)$. Consequently, we finally choose for admissible interval for $\alpha_{\sigma}^{\rho}$ the interval $\mathcal{I}_{\alpha}$ given by:

$$
\mathcal{I}_{\alpha}=\left[0,\left(\min \left(1, r_{\sigma}^{\rho}, \frac{\min \left(\rho_{K}, \tilde{\rho}_{\sigma}\right)}{\rho_{L}} \frac{r_{\sigma}^{\rho}}{r_{\sigma}^{e}}\right)\right)^{+}\right]
$$

The admissible interval for the density is thus $\mathcal{I}_{\rho}$ with

$$
\mathcal{I}_{\rho}=\left\{\rho_{K}+\alpha\left(\rho_{K}-\rho_{M_{\sigma}^{\rho}}\right), \alpha \in \mathcal{I}_{\alpha}\right\} .
$$

Given $\tilde{\rho}_{\sigma}$, the limiting algorithm consists in computing $\rho_{\sigma}$ by projection on $\mathcal{I}_{\rho}$, which yields $\alpha_{\sigma}^{\rho}$; The coefficient $\alpha_{\sigma}^{e}$ is then given by (3.36) and $e_{\sigma}$ is computed from the first relation of (3.31).

We should note that the accuracy of this algorithm depends on the considered variable:

- The approximation for $\rho$, in the absence of limiting procedure, is second order in space.

- Then we derive from this approximation a value for the pressure, using the same weighted average between the neighbouring cells values. In a structured discretization, if no limiting procedure is active, this averaging formula is also the interpolation formula, and thus the face pressure is also given by a second-order formula. On the opposite, for unstructured discretizations, where the interpolation formula (3.29) (more exactly, the analogue of (3.29) written for $p$ ) and the second relation of (3.30) (still replacing $\rho$ by $p$ ) are not the same, the second-order accuracy is lost. - Finally, the internal energy is obtained from the density and the pressure; therefore, its approximation is in general first order, since it only satisfies that the face value lies between the values in the two neighbouring cells. Its computation potentially generates a limiting procedure of the fluxes. In particular, the definition (3.37) of $\mathcal{I}_{\alpha}$ implies that $\alpha_{\sigma}^{\rho}$ vanishes if either $r_{\sigma}^{\rho}$ or $r_{\sigma}^{e}$ is non-positive, i.e. if either $\rho$ or $e$ presents a local extrema.

Several variants of the present scheme may be designed, among which the following:

- As mentioned above, the roles of $\rho$ and $e$ may be reversed, in the sense that one may choose a "limited second order" interpolation for $e$ and $p$, and deduce $\rho$ from these values; to this purpose, one must choose $\kappa=\beta_{\sigma}^{e}$, and start from the non-limited approximation of $e$ instead of the one for $\rho$.

- The present algorithm does not ensure that the value taken for $e$ at the face will lie in-between the second-order approximation and the upwind value. In the case 


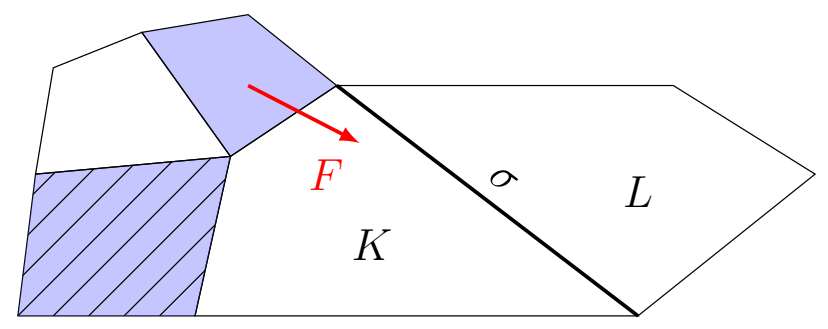

Fig. 3.2. Limiting process - For $\sigma=K \mid L$ and with a constant advection field $\boldsymbol{F}$, the possible cadidates for the upstream cell $M_{\sigma}$ are coloured in blue; the unique opposite cell is hatched.

of one-dimensional or structured discretizations, it may be done by restricting the admissible range for $\beta_{\sigma}^{e}$ to $\beta_{\sigma}^{e} \in\left[\tilde{\beta}_{\sigma}, 1\right]$, where $\tilde{\beta}_{\sigma}$ is the weight which yields for $e_{\sigma}$ the second-order average between $e_{K}$ and $e_{L}$. For uniform meshes, the admissible interval is thus $\beta_{\sigma}^{e} \in[1 / 2,1]$. The results of such a choice would just be an additional limitation of the algorithm.

- Finally, from a theoretical point of view, the upstream cell $M_{\sigma}$ used in the first relation of (3.30) and of (3.31) may be chosen arbitrarily in the mesh, but any reasonable implementation of the algorithm should restrict this choice to the vicinity of the face $\sigma$. Some possible alternatives are given in [34]. In the tests performed here in the remaining of this paper, $M_{\sigma}$ is always the opposite neighbour of the upwind cell $K$ (see Figure 3.2).

3.5. Artificial viscosity. Numerical experiments (see Section 4) show some oscillations at shocks with the upwind scheme developed in [27], probably due to the fact that the artificial viscosity brought by the upwinding behaves as the material velocity only, and not as the celerity of waves; with the MUSCL algorithm, this phenomenon is even enhanced since the numerical diffusion is reduced. To cure this problem, we add some viscosity in the discrete momentum balance equation (while the numerical diffusion in the other equations is left unchanged) and only where it is needed, that is at the shocks. To this purpose, we test here two different methods, inspired from the works [22] and [28] respectively, where the diffusion is evaluated thanks to an a posteriori analysis of the solution.

The aim of this section is to describe the computation of this artificial viscosity, i.e. the parameter $\nu_{\epsilon}^{n+1}$ in Equation (3.4d). The process followed for this computation is to first define a "cell diffusion parameter" $\zeta_{K}^{n+1}$ on each primal cell $K$, and then to deduce the "dual face viscosity" from these cell values. For this latter step, two situations may be encountered:

- The dual face $\epsilon$ is strictly included in a primal cell $K$; in this case, we take $\nu_{\epsilon}^{n+1}=|\epsilon| \zeta_{K}^{n+1}$.

- The dual face $\epsilon$ lies on the boundary of four primal cells (in the MAC case); then we take:

$$
\nu_{\epsilon}^{n+1}=|\epsilon| \frac{1}{4} \sum_{K \in \mathcal{N}(\epsilon)} \zeta_{K}^{n+1}
$$

where $\mathcal{N}(\epsilon)$ is the set of cells adjacent to $\epsilon$.

The remainder of this section is devoted to the description of the computation of the $\left(\zeta_{K}^{n+1}\right)_{K \in \mathcal{M}}$. According to this computation, these parameters are "homogeneous to the space step $h "$ (or, equivalently, the time step, since the CFL number is bounded away from zero and lower than 1 ), in the sense that $\zeta_{K}^{n+1} / h$ (formally) tends neither to zero nor to infinity when the space and time steps tend to zero. Consequently, the artificial diffusion term in $(3.4 \mathrm{~d})$ produces a viscosity which scales as $h^{2}$ in smooth zones of the solution, as in $[22,28]$. However, the scheme proposed here presents two essential differences with these previous works, in order to gain precision at the contact discontinuities while preserving stability: first, artificial diffusion is added 
only in the momentum balance equation (while it is introduced in all the equations in $[22,28])$; second, a first-order upwind discretization is kept in the convection term of the momentum balance equation.

3.5.1. Entropic viscosity. The method, developed in [22], is based on the entropy inequality satisfied by the weak solutions of the system, which reads:

$$
\partial_{t} \eta+\operatorname{div}(\eta u) \leq 0
$$

this inequality becoming an equality in the zones where the solution is smooth and at contact discontinuities. The idea is to compute the numerical diffusion in the momentum balance equation as a function of the entropy production, to introduce an additional numerical dissipation at shocks. We use here the usual physical definition of the entropy:

$$
\eta(p, \rho)=\frac{\rho}{\gamma-1} \log \left(\frac{p}{\rho^{\gamma}}\right)
$$

The first step consists in computing the residual of the discrete entropy equations in every element $K$ of the mesh:

$$
\mathcal{R}_{K}^{n+1}=\frac{1}{\delta t}\left(\eta_{K}^{n+1}-\eta_{K}^{n}\right)+\frac{1}{|K|} \sum_{\sigma \in \mathcal{E}(K)}|\sigma| \eta_{\sigma}^{n} u_{K, \sigma}^{n},
$$

where $\eta_{\sigma}^{n}$ stands for a centered approximation of the entropy at the faces $\sigma$. Then we compute a tentative diffusion parameter by:

$$
\tilde{\tilde{\zeta}}_{K}^{n+1}=c_{E} \rho_{K}^{n+1} h_{K}\left|\mathcal{R}_{K}^{n+1}\right| .
$$

where $h_{K}$ is the diameter of the cell $K$ and $c_{E}$ is a calibration parameter. Note that $\mathcal{R}_{K}^{n+1}$ is a formal discretization of $\partial_{t} \eta+\operatorname{div}(\eta u)$, and thus is a quantity formally independent of the space and time steps; consequently, $\tilde{\tilde{\zeta}}_{K}^{n+1}$ scales as $h_{K}$. Then this parameter is limited to the (range of the) diffusion generated by the first-order upwinding of the convection operator. For any face $\sigma$ of the primal mesh adjacent to a cell $L$, this latter reads $\zeta_{\sigma}^{n+1}=\left|\rho_{\sigma}^{n} u_{L, \sigma}^{n}\right| / 2$, with $\rho_{\sigma}^{n}$ the face density used in the mass balance equation. We then define a maximum value for the diffusion parameter by:

$$
\zeta_{\max , K}^{n+1}=c_{\max } \max \left(\left(\zeta_{\sigma}^{n+1}\right)_{\sigma \in \overline{\mathcal{E}}(K)}\right)
$$

where $c_{\max }$ is once again a calibration parameter and $\overline{\mathcal{E}}(K)$ stands for a set of faces located in the vicinity of $K$, whichs includes at least $\mathcal{E}(K)$. In the context of the present applications, this set is in fact much larger, since it is composed of the faces of the 3 left and 3 right cells to $K$ in one dimension, and for structured 2D discretizations, the faces of the cells of a $7 \times 7$ patch centered on $K$. Then we obtain a second tentative diffusion parameter by:

$$
\tilde{\zeta}_{K}^{n+1}=\min \left(\tilde{\tilde{\zeta}}_{K}^{n+1}, \zeta_{\max , K}^{n+1}\right)
$$

Finally, $\zeta_{K}^{n+1}$ is computed as a weighted average of the parameters $\left(\tilde{\zeta}_{L}^{n+1}\right)_{L \in \mathcal{M}}$ over a patch around $K$. In one dimension, this patch includes the left and right cells of $K$ and $K$ itself, and the weight is $2 / 3$ for $K$ and $1 / 3$ for the other cells. For structured discretizations in two dimensions, we use a $3 \times 3$ patch centered on $K$, the weight is $8 / 9$ for $K$ and $1 / 9$ for the other cells.

3.5.2. WLR viscosity. The second method is based on [28]. We first briefly recall the ideas developped in this work, for a generic conservation law of unknown $w$ and flux $f$ :

$$
\partial_{t} w+\operatorname{div} \boldsymbol{f}(w)=0
$$


A weak solution of $(3.40)$ is a function $w \in L^{1}(0, T ; \Omega)$ such that:

$$
\begin{aligned}
\mathcal{W}(w, \phi)=\int_{0}^{T} \int_{\Omega}\left[w(\boldsymbol{x}, t) \partial_{t} \phi(\boldsymbol{x}, t)+\boldsymbol{f}(\boldsymbol{x}, t)\right. & \cdot \boldsymbol{\nabla} \phi(\boldsymbol{x}, t)] \mathrm{d} \boldsymbol{x} \mathrm{d} t \\
& +\int_{\Omega} w(\boldsymbol{x}, 0) \phi(\boldsymbol{x}, 0) \mathrm{d} \boldsymbol{x}=0,
\end{aligned}
$$

for all test functions $\phi \in C_{0}^{1}(\Omega \times[0, T))$. This identity is used in [28] to build, on the basis of a discrete solution $w_{h}$ obtained by a finite difference method, a measure of the local regularity of the solution. The discrete solution is identified to a function of time and space, specific test functions $\phi$ (one per cell, let us say $\left(\phi_{K}\right)_{K \in \mathcal{M}}$ to keep notations consistent with the rest of the present paper) are defined, and the quantities $\left(\mathcal{W}\left(w_{h}, \phi_{K}\right)\right)_{K \in \mathcal{M}}$ are used to track the discontinuities. On their basis, a stabilizing diffusion is then introduced in the scheme.

Here, we use an adaptation of this strategy for the Euler equations and a finite volume scheme. First, we do not compute the residual $\mathcal{W}$ for each equation, but just for the mass balance:

$$
\begin{array}{r}
\mathcal{W}(\rho, \boldsymbol{u}, \phi)=\int_{0}^{T} \int_{\Omega}\left[\rho(\boldsymbol{x}, t) \partial_{t} \phi_{t}(\boldsymbol{x}, t)+\rho(\boldsymbol{x}, t) \boldsymbol{u}(\boldsymbol{x}, t) \cdot \boldsymbol{\nabla} \phi(\boldsymbol{x}, t)\right] \mathrm{d} \boldsymbol{x} \mathrm{d} t \\
+\int_{\Omega} \rho(\boldsymbol{x}, 0) \phi(\boldsymbol{x}, 0) \mathrm{d} \boldsymbol{x} .
\end{array}
$$

As for the finite difference scheme treated in [28], we identify the discrete solution to piecewise functions. We thus define:

$$
\begin{aligned}
\rho_{\Delta}(\boldsymbol{x}, t) & =\sum_{n=0}^{N-1} \sum_{K \in \mathcal{M}} \rho_{K}^{n} \mathcal{X}_{K}(\boldsymbol{x}) \mathcal{X}_{\left(t_{n}, t_{n+1}\right)}(t), \\
\boldsymbol{u}_{\Delta}(\boldsymbol{x}, t) & =\sum_{n=0}^{N-1} \sum_{K \in \mathcal{M}} \boldsymbol{u}_{K}^{n} \mathcal{X}_{K}(\boldsymbol{x}) \mathcal{X}_{\left(t_{n}, t_{n+1}\right)}(t),
\end{aligned}
$$

where $\mathcal{X}_{K}$ and $\mathcal{X}_{\left(t_{n}, t_{n+1}\right)}$ stand for the characteristic functions of the cell $K$ and the interval $\left(t_{n}, t_{n+1}\right)$ and $\boldsymbol{u}_{K}$ is an interpolation of the velocity on the primal mesh:

$$
\forall K \in \mathcal{M}, \quad \boldsymbol{u}_{K}=\frac{1}{|K|} \sum_{\sigma \in \mathcal{E}(K)}\left|D_{K, \sigma}\right| \boldsymbol{u}_{\sigma} .
$$

The next step is to introduce a set of local polynomials $\left(\phi_{K}^{n}\right)$, for every $K \in \mathcal{M}$ and for $0 \leq n \leq N-1$, to be used as test functions. We postpone the exact definition of these polynomials for a while, and only state here the approximation property that they have to satisfy for the subsequent theory to hold. For any $\phi \in C_{0}^{1}(\Omega \times[0, T])$, we suppose that there exists $\left(\beta_{K}^{n}\right)_{K \in \mathcal{M}, 0 \leq n \leq N-1} \subset \mathbb{R}$ such that:

$$
\phi(\boldsymbol{x}, t)=\sum_{K \in \mathcal{M}} \sum_{n=0}^{N-1} \beta_{K}^{n} \phi_{K}^{n}(\boldsymbol{x}, t)+\mathcal{O}\left(\Delta^{2}\right),
$$

where $\Delta=\max (h, \delta t)$. If we suppose that the test functions $\left(\phi_{K}^{n}\right)$ are local in the sense that their integral behaves like $\delta t h^{d}$ (since the measure of their support also behaves like $\left.\delta t h^{d}\right)$, this condition ensures that

$$
\mathcal{W}\left(\rho_{\Delta}, \boldsymbol{u}_{\Delta}, \phi\right)=\sum_{K \in \mathcal{M}} \sum_{n=0}^{N-1}\left(\beta_{K}^{n} \mathcal{W}_{K}^{n}+\mathcal{O}\left(\Delta^{d+3}\right)\right),
$$

where the weak local residual (WLR), $\mathcal{W}_{K}^{n}$, takes the following expression:

$$
\mathcal{W}_{K}^{n}=\int_{0}^{T} \int_{\Omega} \rho_{\Delta}(\boldsymbol{x}, t) \partial_{t} \phi_{K}^{n}(\boldsymbol{x}, t)+\rho_{\Delta}(\boldsymbol{x}, t) \boldsymbol{u}_{\Delta}(\boldsymbol{x}, t) \cdot \boldsymbol{\nabla} \phi_{K}^{n}(\boldsymbol{x}, t) \mathrm{d} \boldsymbol{x} \mathrm{d} t
$$


In the one-dimensional case, it is proven in [28] that, under the assumption (3.41), these weak local residuals have the following properties:

$$
\left|\mathcal{W}_{K}^{n}\right| \text { behaves as } \mid \begin{array}{ll}
\Delta, & \text { near shock waves, } \\
\Delta^{\alpha}, & 1<\alpha \leq 2, \text { near contact waves, } \\
\Delta^{3}, & \text { in smooth regions. }
\end{array}
$$

These results are not proven in two and three dimensions; however the same behaviour for $\left|\mathcal{W}_{K}^{n}\right| / \Delta^{d-1}$ is observed on numerical tests. These residuals are used to define a tentative diffusion coefficient by:

$$
\tilde{\zeta}_{K}^{n+1}=c_{m} \frac{1}{\delta t \Delta^{d-1}}\left|\mathcal{W}_{K}^{n+1}\right|
$$

with $c_{m}$ a calibration parameter. Finally, $\zeta_{K}^{n+1}$ is computed as a weighted average of the parameters $\left(\tilde{\zeta}_{L}^{n+1}\right)_{L \in \mathcal{M}}$ over a patch around $K$, exactly in the same way as for the entropic viscosity.

In the applications presented in Section 4 below, we use for the polynomials $\left(\phi_{K}^{n}\right)_{K \in \mathcal{M}, 0 \leq n \leq N-1}$ the same definition based on B-splines as in [28].

4. Numerical tests. We present in this section numerical tests to assess the behaviour of the scheme. We first address the accuracy of the MUSCL technique by performing a convergence study on a regular solution, posed on a two-dimensional domain. Then we turn to Riemann problems, first in one then in two space dimensions. In one dimension, the first test is a pure contact discontinuity, and allows to check the importance of a flux construction keeping the velocity and the pressure constant in such a situation. Then we check the accuracy of the MUSCL interpolation and artificial viscosity techniques on several 1D numerical test cases. A convergence rate analysis is performed, to compare upwind and MUSCL interpolations in the case of a shock solution. We then turn to two classical two-dimensional benchmarks for Euler solvers, featuring reflection phenomena, namely the so-called Mach 3 wind tunnel with step and the double Mach reflection. Since all these tests use the MAC space discretization, we complete the study by computing a high speed inviscid flow around a cylinder with the Rannacher-Turek space discretization. For all the computations, the fluid obeys the equation of state (1.1d) with $\gamma=1.4$.

4.1. Convergence study for regular solutions. We begin with a test where the velocity and pressure are constant at $t=0$, and the density and internal energy are smooth functions of space such that, to satisfy the equation of state, the product $\rho e$ is constant. Let the computational domain be the square $\Omega=(-1.2,2)^{2}$ and $\zeta$ be given by $\zeta=x_{1}^{2}+x_{2}^{2}$. The initial data is:

$$
\boldsymbol{u}=\left[\begin{array}{l}
1 \\
1
\end{array}\right], p=1, \rho=1+64 \zeta^{3}(1-\zeta)^{3} \text { if } \zeta \leq 1, \rho=1 \text { otherwise, } e=\frac{1}{(\gamma-1) \rho}
$$

The time interval is $(0,0.8)$ and, within this time interval and with suitable boundary conditions on the frontier of the domain, the velocity and the pressure remain constant and the density and internal energy are just transported; thus, the solution at $t=0.8$ is obtained by a translation of vector $(0,8,0.8)^{t}$ of the initial density and internal energy fields.

Computations are performed on a DELL Latitude E 7470 portable PC. The total CPU time for a mesh of 640000 cells and 100 time steps is 180 s, which yields a CPU time per cell and time step slightly lower than $310^{-6} \mathrm{~s}$; this performance measure is obtained using the $\mathrm{CALIF}^{3} \mathrm{~S}$ CFD code [10] developed at the French Institut de Radioprotection et de Sûreté Nucléaire, designed to deal with unstructured meshes and can be strongly reduced (up to a factor near than 10) using a software dedicated to uniform structured meshes only. 
First of all, we observe that, thanks to the construction of the fluxes, the scheme keeps the pressure and velocity constant, both for the first order upwind and MUSCLlike variants. The density profiles obtained at $t=0.8$ with an $n \times n$ regular grid with $n=100$ and $\delta t=h / 8$ are shown on Figure 4.1 , and the discrete $L^{1}$ norm of the density error for $n=100,200,400,800$ and still $\delta t=h / 8$ is plotted on Figure 4.2. This latter norm is defined by:

$$
\left\|\epsilon_{\rho}\right\|_{L_{\mathcal{M}}^{1}}=\sum_{K \in \mathcal{M}}|K|\left|\rho_{K}-\rho\left(\boldsymbol{x}_{K}\right)\right| \text {, with } \boldsymbol{x}_{K} \text { the mass center of } K \text {. }
$$

We observe that the MUSCL scheme is much more precise, but still first-order, which we explain by the fact that the time discretization is still first-order. To check this fact, we implement a second order Runge-Kutta scheme (also called Heun scheme), which reads:

$$
\begin{aligned}
& \boldsymbol{W}^{n+\frac{1}{3}}=\mathcal{S}\left(\boldsymbol{W}^{n}\right) \\
& \boldsymbol{W}^{n+\frac{2}{3}}=\mathcal{S}\left(\boldsymbol{W}^{n+\frac{1}{3}}\right) \\
& \boldsymbol{W}^{n+1}=\frac{1}{2}\left(\boldsymbol{W}^{n}+\boldsymbol{W}^{n+\frac{2}{3}}\right),
\end{aligned}
$$

where $\boldsymbol{W}=(\rho, e, p, \boldsymbol{u})$ and the relation $\boldsymbol{W}^{n+\frac{1}{3}}=\mathcal{S}\left(\boldsymbol{W}^{n}\right)$ means that the left-hand side is obtained by applying the standard first-order in time explicit scheme to an initial data given by $\boldsymbol{W}^{n}$. Note that, by construction, this scheme also keeps the pressure and velocity constant if so does the first-order explicit scheme. Results are reported also on Figures 4.1 and 4.2, and we observe that we now obtain a second order convergence. However, the same test but with discontinuous initial density and internal energy shows that the Runge-Kuntta time discretization adds an excessive diffusion to the scheme, and that the full second-order scheme turns to be, for such a solution, less accurate that the scheme combining a first-order time discretization and a MUSCL-like expression of the fluxes. Since we address shock solutions in the remainder of the paper, we thus keep this latter variant.

For solutions where the velocity varies, the proposed scheme is in any case no better than first-order because of the standard upwind space discretization of the momentum convection fluxes in the momentum balance equation. Here again, we build a second-order scheme, and perform tests on a moving smooth barotropic vortex. This second-order scheme is obtained thanks to the following two modifications, with the following associated consequences:

- First, a second-order approximation of the face velocity in the above-mentioned momentum fluxes is needed. Unfortunately, doing so, the non-negativity of the corrective term in the internal energy balance is no-more ensured, and stability properties are thus lost, at least from a theoretical point of view. Note that the sign of this term may be recovered if a viscosity term is present.

- Second, a second-order time-discretization as (4.1) must be implemented. Note that, in Relation (4.1), the scheme $\mathcal{S}$ is the standard explicit scheme, which means in particular that the use of the end-of-step pressure in the momentum balance equation is forbidden. However, we observed in [27] for the explicit first-order scheme that using $p^{n}$ in the momentum balance may have as consequence the appearance of non-entropic discontinuities in rarefaction waves; whether this is still true with a Runge-Kutta discretization is unclear.

From these results, the extension to second-order that we obtain seems to be a good candidate to solve the compressible Navier-Stokes equations (especially, if one wants to keep explicit steps and thus an explicit discretization of the diffusion terms, for small viscosity flows as addressed in Large Eddy Simulation applications), but the inviscid case should deserve further work. Note however that, as already mentioned, the full second order scheme always induces an excessive diffusion for shock solutions so that in this case, the variant which is studied here should be preferred. 


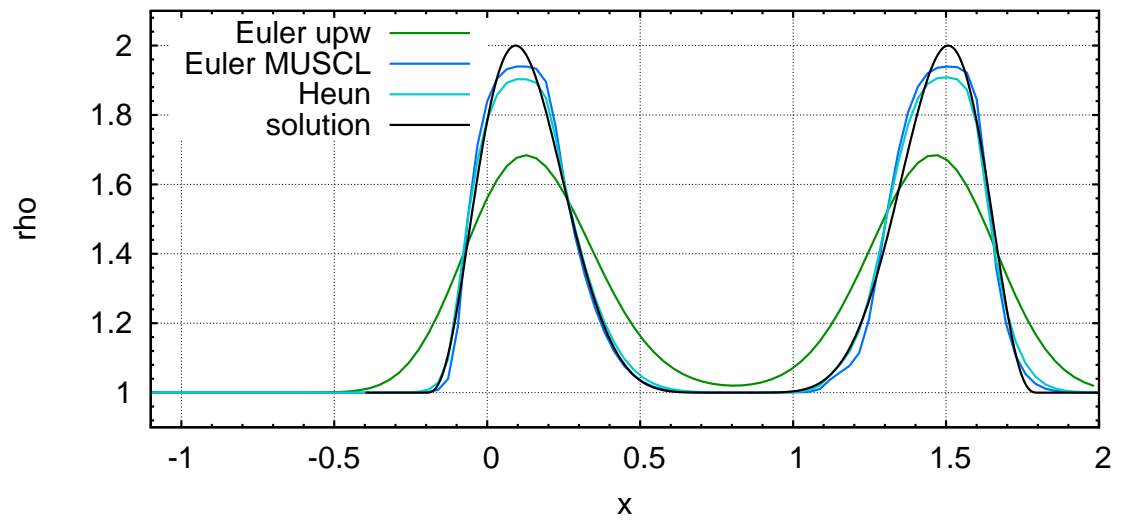

FIG. 4.1. A 2D Regular problem with constant pressure and velocity $-h=0.032$ and $\delta t=$ $h / 8$ - Density along the line $x_{2}=0.8$ at $t=0.8$ obtained with three different schemes: firstorder time discretization (Euler scheme) and first order upwind convection scheme, first-order time discretization and MUSCL convection scheme and Heun time discretization and MUSCL convection scheme.

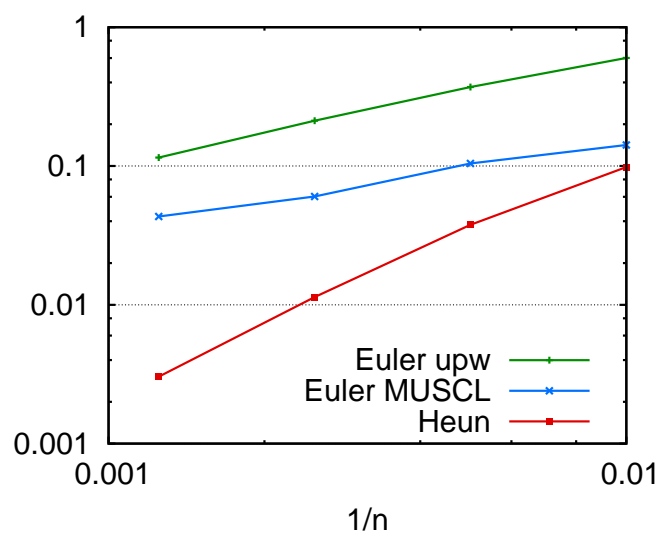

FIG. 4.2. A 2D Regular problem with constant pressure and velocity $-L^{1}$ errors on the density at $t=0.8$ obtained with three different schemes: first-order time discretization (Euler scheme) and first order upwind convection scheme, first-order time discretization and MUSCL convection scheme and Heun time discretization and MUSCL convection scheme. The space step is $h=3.2 / n$ with $n=100,200,400,800$ and the time step is $\delta t=h / 8$.

4.2. One dimensional Riemann problems. This section is devoted to the computation of one-dimensional Rieman problems. In all the tests, the computational domain is $\Omega=(0,1)$ and the initial discontinuity is located at $x=0.5$.

Single contact discontinuity wave - First of all, we give a numerical evidence of the necessity of a correlation between the face approximation of the density and the internal energy. To this purpose, we compute a Riemann problem consisting in a single contact discontinuity wave travelling to the right of the domain. It corresponds to the following initial conditions:

$$
\text { left state: }\left[\begin{array}{l}
\rho_{L}=14.282 \\
u_{L}=8.6898 \\
p_{L}=1691.6
\end{array}\right] ; \quad \text { right state: }\left[\begin{array}{l}
\rho_{R}=31.043 \\
u_{R}=8.6898 \\
p_{R}=1691.6
\end{array}\right] \text {. }
$$

The pressure fields obtained, at $t=0.02$, respectively with and without a correlation between the approximation of the density and of the internal energy at faces of the cells, are shown on Figure 4.3. The computation referred to as "non-correlated approximation" is performed by applying the same interpolation/limiting procedure used for the density bluntly to the internal energy, without imposing that the product 


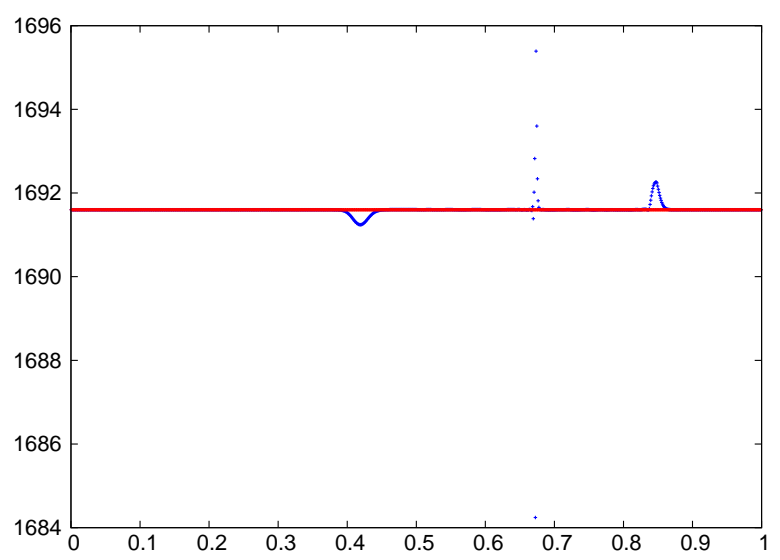

FIG. 4.3. A 1D Riemann problem: single contact discontinuity $-h=0.001$ and $\delta t=h / 40-$ Pressure at $t=0.02$. Results obtained with a correlated (resp. non-correlated) face approximation for $\rho$ and $e$ are drawn in red (resp. in blue). The analytical solution is the same as the discrete solution obtained with the correlated approximation (non-visible black line).

$\rho e$ at the face be an interpolation of $\rho e$ at the two neighbour cells. As one can see, this approximation generates oscillations of the pressure at the contact discontinuity; we even observe in our computations that these oscillations tend to get worse with time. In addition, pressure variations appear at the locations of zero-amplitude 1-shock and 3 -shock waves. On the opposite, the proposed scheme yields a constant pressure with respect to time and space, as in the continuous solution.

Two classical Riemann problems - We will now compare the upwind and the MUSCL schemes on two Riemann problems classically used in the literature, namely Test 4 and Test 5 from [39, Chapter 4]. In Test 4, the left and right states are:

$$
\text { left state: }\left[\begin{array}{c}
\rho_{L}=1 \\
u_{L}=0 \\
p_{L}=0.01
\end{array}\right] ; \quad \text { right state: }\left[\begin{array}{c}
\rho_{R}=1 \\
u_{R}=0 \\
p_{R}=100
\end{array}\right] .
$$

The solution consists of a shock travelling to the left and a rarefaction wave travelling to the right, separated by the contact discontinuity. We first evaluate the stability of the scheme, by performing runs for increasing (and constant for each run) time steps, until the computation blows up; for $h=0.001$, strong oscillations are observed for $\delta t=h / 18$ and the computation fails for $\delta t=h / 17$; since the maximal celerity of waves (estimated from the analytical solution) is close to 17 , the CFL number is close to 1.

This stability limit is obtained without artificial viscosity; adding such a term reduces the stability domain. Note hovever that, since the artificial diffusion is limited by the viscosity generated by the first-order upwind scheme, the stability domain still keeps the form $\delta t \leq C h$ (and not $\delta t \leq C h^{2}$, which would be characteristic of a viscosity constant (in order of magnitude) with respect to the space step). Results obtained at $t=0.035$ with $h=0.001$ and $\delta t=h / 30$ are reported on Figure 4.4. As seen on the internal energy and density profiles, the numerical diffusion at the contact discontinuity is drastically reduced by the MUSCL approximation. At the shock, the results of the upwind and the MUSCL scheme look similar: on one hand, the compressive effect of the shock prevents the upwind scheme to be too dissipative, and, on the other hand, the numerical dissipation introduced by the limiting procedure in the MUSCL scheme seems to be sufficient.

In Test 5 , the initial conditions are:

$$
\text { left state: }\left[\begin{array}{l}
\rho_{L}=5.99924 \\
u_{L}=19.5975 \\
p_{L}=460.894
\end{array}\right] ; \quad \text { right state: }\left[\begin{array}{c}
\rho_{R}=5.99242 \\
u_{R}=-6.19633 \\
p_{R}=46.0950
\end{array}\right] .
$$



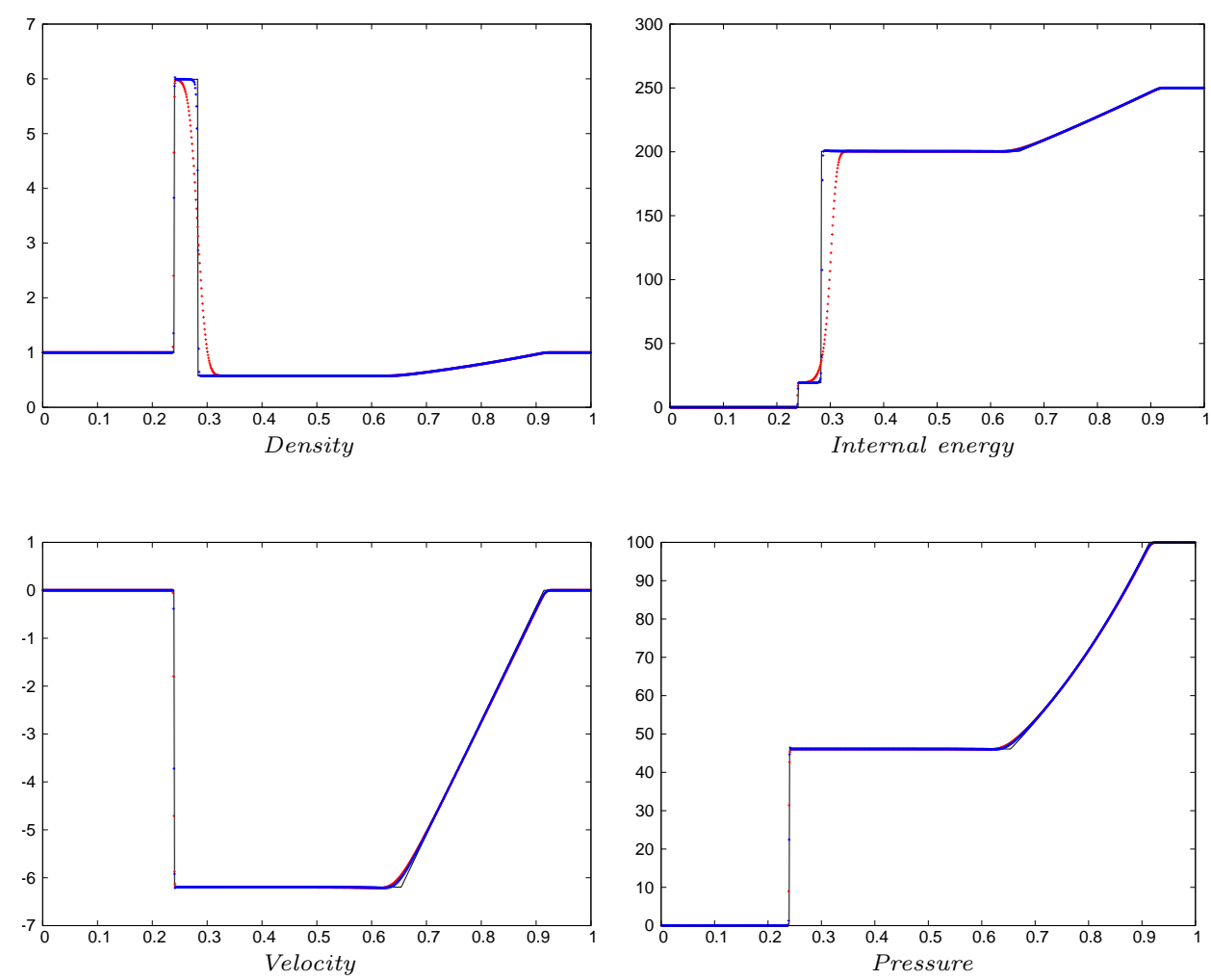

FIG. 4.4. A $1 D$ Riemann problem: Test 4 of [39, Chapter 4] $-h=0.001$ and $\delta t=h / 30-$ Results at $t=0.035$. The upwind and MUSCL solutions are drawn in red and blue respectively, the analytical solution corresponds to the black line.

In this test, the genuinely non-linear waves are two shocks travelling to the left. The numerical stability analysis shows that, without artificial viscosity and for $h=0.001$, the scheme blows up for $\delta t \simeq h / 29$ (while the greatest wave celerity is close to 30 in the left state). Results obtained at $t=0.035$ with $h=0.001$ and $\delta t=h / 90$ are reported on Figure 4.5. One may observe on the density and the pressure some overshoots at the 3 -shock with the upwind scheme; this phenomenon is strengthened with the MUSCL algorithm (results are not shown here). This problem is completely cured by the WLR viscosity introduced in Section 3.5.2, with $c_{m}=2$ in Relation (3.43).

A convergence study for a shock solution - In addition, we perform a convergence study, successively dividing by two the space and time steps (so keeping the CFL number constant). We use the same test as in [27], i.e. Test 3 in [39, Chapter 4]. The left and right states are given by:

$$
\text { left state: }\left[\begin{array}{c}
\rho_{L}=1 \\
u_{L}=0 \\
p_{L}=1000
\end{array}\right] ; \quad \text { right state: }\left[\begin{array}{c}
\rho_{R}=1 \\
u_{R}=0 \\
p_{R}=0.001
\end{array}\right] \text {. }
$$

The solution combines a rarefaction wave travelling to the left, the contact discontinuity and a shock wave travelling to the right. The differences between the computed and analytical solution at $t=0.012$, measured in $\mathrm{L}^{1}(\Omega)$ norm, are reported in the following table:

\begin{tabular}{|c|ccccc|}
\hline space step & $h_{0}=0.001$ & $h_{0} / 2$ & $h_{0} / 4$ & $h_{0} / 8$ & $h_{0} / 16$ \\
\hline$\|\rho-\bar{\rho}\|_{\mathrm{L}^{1}(\Omega)}$ & 0.0108 & 0.0058 & 0.0025 & 0.0012 & 0.0007 \\
\hline convergence rate, $\rho$ & - & 0.91 & 1.22 & 1.06 & 0.78 \\
\hline$\|p-\bar{p}\|_{\mathrm{L}^{1}(\Omega)}$ & 1.2827 & 0.6734 & 0.3316 & 0.1800 & 0.1044 \\
\hline convergence rate, $p$ & - & 0.93 & 1.02 & 0.88 & 0.79 \\
\hline
\end{tabular}



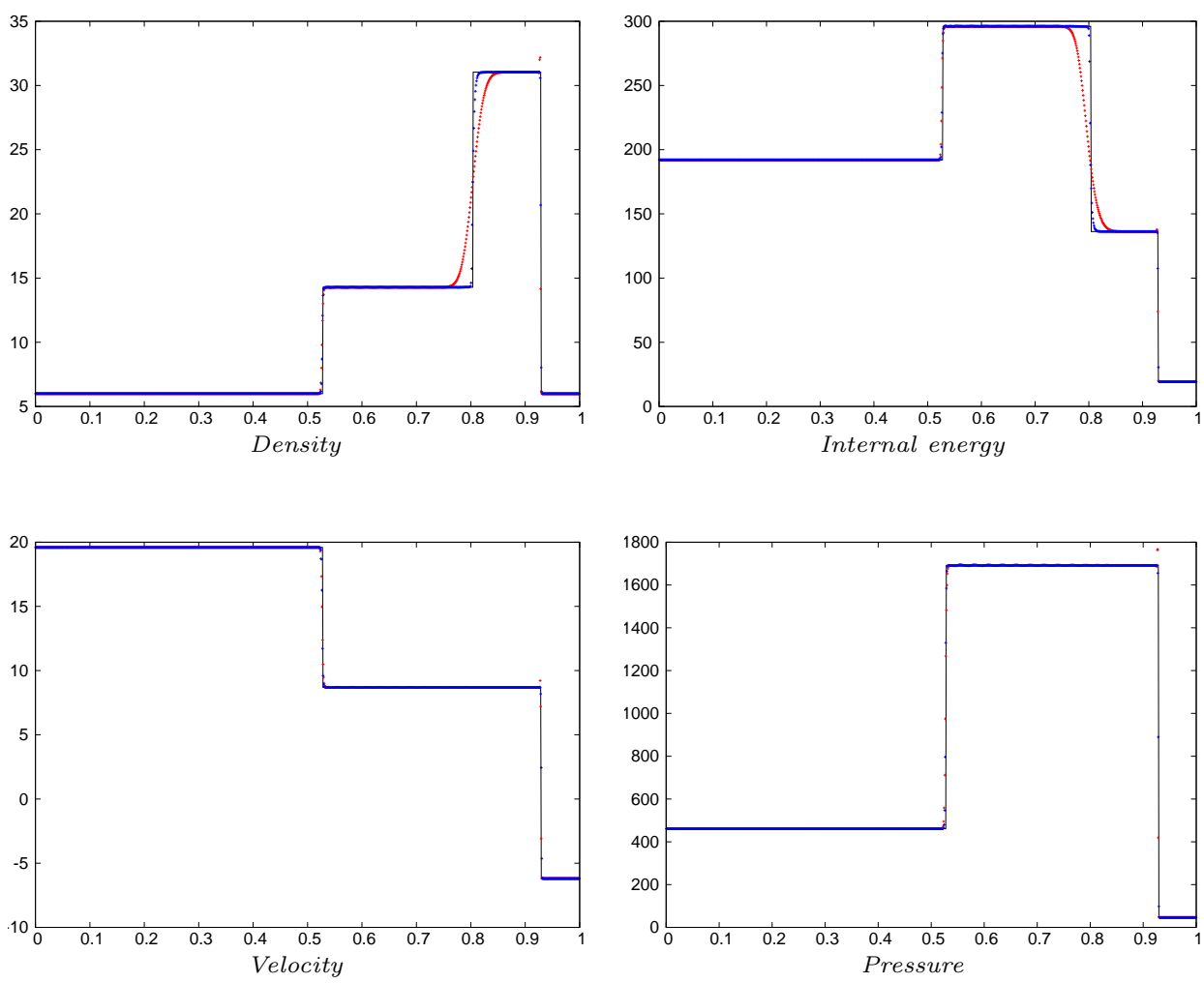

FIG. 4.5. A $1 D$ Riemann problem: Test 5 of [39, Chapter 4] $-h=0.001$ and $\delta t=h / 90-$ Results at $t=0.035$. The upwind and MUSCL (with artificial viscosity) solutions are drawn in red and blue respectively, the analytical solution corresponds to the black line.

We recall the results obtained with the upwind scheme:

\begin{tabular}{|c|ccccc|}
\hline space step & $h_{0}=0.001$ & $h_{0} / 2$ & $h_{0} / 4$ & $h_{0} / 8$ & $h_{0} / 16$ \\
\hline$\|\rho-\bar{\rho}\|_{\mathrm{L}^{1}(\Omega)}$ & 0.0651 & 0.0455 & 0.0310 & 0.0217 & 0.0153 \\
\hline convergence rate, $\rho$ & - & 0.52 & 0.55 & 0.51 & 0.5 \\
\hline$\|p-\bar{p}\|_{\mathrm{L}^{1}(\Omega)}$ & 1.87 & 1.05 & 0.530 & 0.284 & 0.164 \\
\hline convergence rate, $p$ & - & 0.83 & 0.99 & 0.9 & 0.79 \\
\hline
\end{tabular}

As one can see, the convergence rate is improved by the MUSCL interpolation. Indeed, for variables which are not constant through contact discontinuities, the convergence rate is now close to 1 . For the other variables, it is slightly improved.

The symmetrical double-shock - To conclude the part devoted to one-dimensional tests, we address a pathological case, where the initial data consists in opposite initial velocities, the density and pressure being constant all over $\Omega$. Precisely speaking, we take:

$$
\text { left state: }\left[\begin{array}{l}
\rho_{L}=5.99924 \\
u_{L}=19.5975 \\
p_{L}=460.894
\end{array}\right] ; \quad \text { right state: }\left[\begin{array}{c}
\rho_{R}=5.99924 \\
u_{R}=-19.5975 \\
p_{R}=460.894
\end{array}\right] .
$$

The analytical solution consists in two shocks, travelling with the same velocity to the left and the right respectively, which separate the left and right initial states from a constant state where the fluid is at rest; the contact discontinuity is stationary, located at $x=0.5$ and of zero amplitude. Results obtained at $t=0.035$ with $h=0.001$ and $\delta t=h / 60$ are reported on Figure 4.6. This test case is particulary interesting because the dual convection fluxes vanish in the intermediate state. Consequently, the upwind scheme (solution in red on the figure) does not bring any 
numerical viscosity in the central zone (since this viscosity is proportional to $\left|F_{\sigma, \epsilon}\right| / 2$ ), and spurious oscillations appear. The WLR viscosity, with $c_{m}=3$, allows to drastically reduce this phenomenon. However, it also generates artificial variations at the contact discontinuity for the (possibly) discontinuous variables; the other ones are not affected.

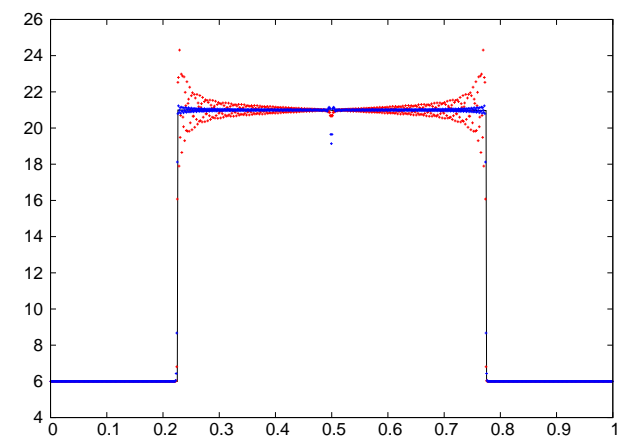

FIG. 4.6. A $1 D$ Riemann problem: the "symetrical double shock" $-h=0.001$ and $\delta t=h / 60-$ Density at $t=0.035$. Upwind solution without (red) and with (blue) WLR viscosity.

4.3. Two-dimensional Riemann problems. The computational domain is $\Omega=(-0.5,0.5)^{2}$ and is split in four quadrants $\Omega_{1}=(0,0.5)^{2}, \Omega_{2}=(-0.5,0) \times(0,0.5)$, $\Omega_{3}=(-0.5,0)^{2}, \Omega_{4}=(0,0.5) \times(-0.5,0)$. A the initial time, the solution is constant over each quadrant, and the constant states are chosen so that the solution to the four Riemann problems associated with each interface of the quadrants consists in a single wave. Nineteen possible configurations, yielding genuinely different solutions, heve been reported in the literature (see e.g. $[37,30,29]$ ); we compute here only three of them. All the computations of this section are performed with the MAC space discretization.

Configurations 5 and $\mathbf{6}$-MUSCL interpolation is primarily used to improve precision at contact discontinuity lines. To illustrate this effect, we address Configurations referred to as 5 and 6 in [30], where the chosen discontinuities are such that they generate two-dimensional contact discontinuity waves (i.e. the pressure and the normal velocity are constant across the discontinuity, and the internal energy, the density and the tangential velocity jump). For Configuration 5 , the initial condition is:

$$
\begin{array}{c|c|c}
\Omega_{2}: \quad\left[\begin{array}{c}
\rho_{2}=2 \\
p_{2}=1 \\
u_{2}=-0.75 \\
v_{2}=0.5
\end{array}\right] & \Omega_{1}:\left[\begin{array}{c}
\rho_{1}=1 \\
p_{1}=1 \\
u_{1}=-0.75 \\
v_{1}=-0.5
\end{array}\right] \\
\Omega_{3}: \quad\left[\begin{array}{c}
\rho_{3}=1 \\
p_{3}=1 \\
u_{3}=0.75 \\
v_{3}=0.5
\end{array}\right] & \Omega_{4}:\left[\begin{array}{c}
\rho_{4}=3 \\
p_{4}=1 \\
u_{4}=0.75 \\
v_{4}=-0.5
\end{array}\right]
\end{array}
$$

For Configuration 6, we have: 

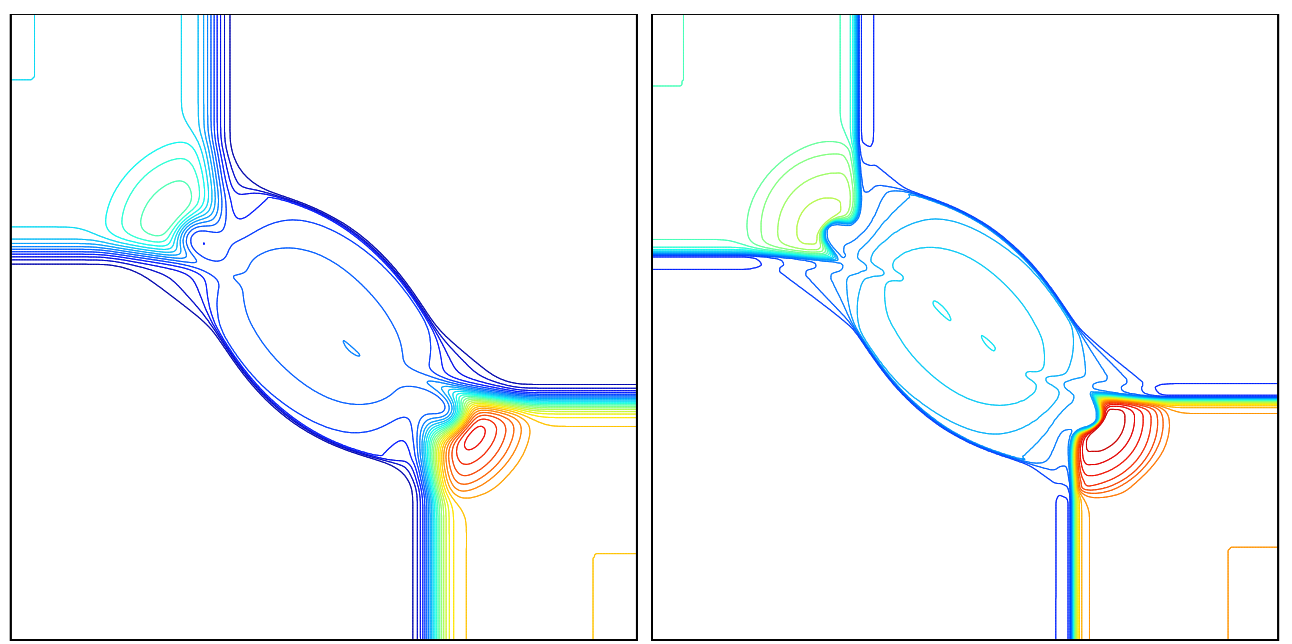

FIG. 4.7. A two-dimensional Riemann problem: Configuration 5 in [30] - Comparison of the upwind (left) and MUSCL (right) schemes $-h=1 / 400$ and $\delta t=h / 10-$ Density at $t=0.23$.
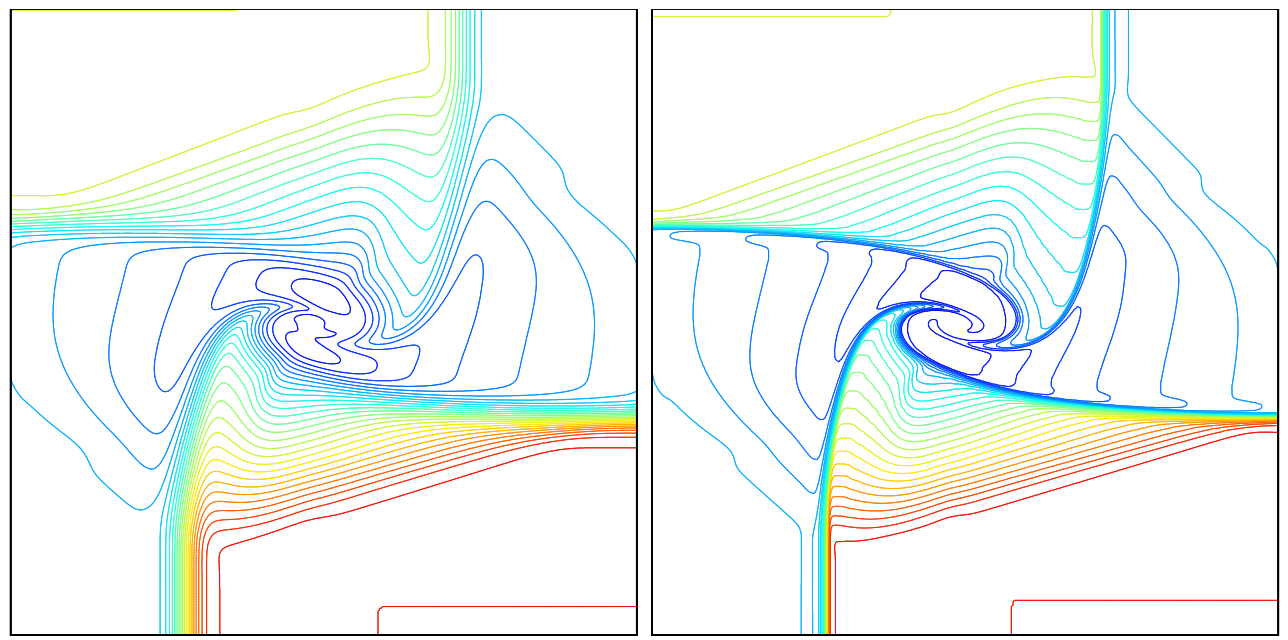

FIG. 4.8. A two-dimensional Riemann problem: Configuration 6 in [30]-Comparison of the upwind (left) and MUSCL (right) schemes $-h=1 / 400$ and $\delta t=h / 10-$ Density at $t=0.3$.

$$
\begin{array}{c|c}
\Omega_{2}: \quad\left[\begin{array}{c}
\rho_{2}=2 \\
p_{2}=1 \\
u_{2}=0.75 \\
v_{2}=0.5
\end{array}\right] & \Omega_{1}:\left[\begin{array}{c}
\rho_{1}=1 \\
p_{1}=1 \\
u_{1}=0.75 \\
v_{1}=-0.5
\end{array}\right] \\
\hline \Omega_{3}: \quad\left[\begin{array}{c}
\rho_{3}=1 \\
p_{3}=1 \\
u_{3}=-0.75 \\
v_{3}=0.5
\end{array}\right] & \Omega_{4}:\left[\begin{array}{c}
\rho_{4}=3 \\
p_{4}=1 \\
u_{4}=-0.75 \\
v_{4}=-0.5
\end{array}\right]
\end{array}
$$

The final time is $t=0.23$ for Configuration 5 and $t=0.3$ for Configuration 6 . Results obtained with the upwind and MUSCL schemes at the end of the computation, with a $400 \times 400$ grid and with $\delta t=1 /(10 \times 400)$, are reported on Figures 4.7 and 4.8 respectively. Solutions are consistent with the results reported in the litterature and, as we expect, the slip lines are sharper with the MUSCL interpolation.

Configuration 4 - We now turn to a test case where the elementary waves are shockwaves, to evidence the properties of entropic and WLR viscosities. The initial states are now given by: 


$$
\begin{array}{cc}
\Omega_{2}:\left[\begin{array}{c}
\rho_{2}=0.5065 \\
p_{2}=0.35 \\
u_{2}=0.8939 \\
v_{2}=0
\end{array}\right] & \Omega_{1}:\left[\begin{array}{c}
\rho_{1}=1.1 \\
p_{1}=1.1 \\
u_{1}=0 \\
v_{1}=0
\end{array}\right] \\
\hline \Omega_{3}: \quad\left[\begin{array}{c}
\rho_{3}=1.1 \\
p_{3}=1.1 \\
u_{3}=0.8939 \\
v_{3}=0.8939
\end{array}\right] & \Omega_{4}:\left[\begin{array}{c}
\rho_{4}=0.5065 \\
p_{4}=0.35 \\
u_{4}=0 \\
v_{4}=0.8939
\end{array}\right]
\end{array}
$$

Results obtained at $t=0.3$ on a $400 \times 400$ grid with $\delta t=1 /(10 \times 400)$ are reported on Figure 4.9. The first-order upwind scheme yields a solution (Figure 4.9, top-right) with spurious oscillations in the downstream section of the top and right shock, in the area where the fluid is at rest. This is caused by the lack of numerical dissipation of our scheme, because the dissipation produced by the upwind interpolation vanishes with the velocity. To cure this problem, we first add a constant artificial viscosity (Figure 4.9, top-right). We also plot the results obtained using the WLR and entropic viscosities (Figure 4.9, middle and bottom line, respectively). As one can see, they correctly pick up shocks and reduce oscillations inside the subsonic area surrounded by the sonic shocks. 


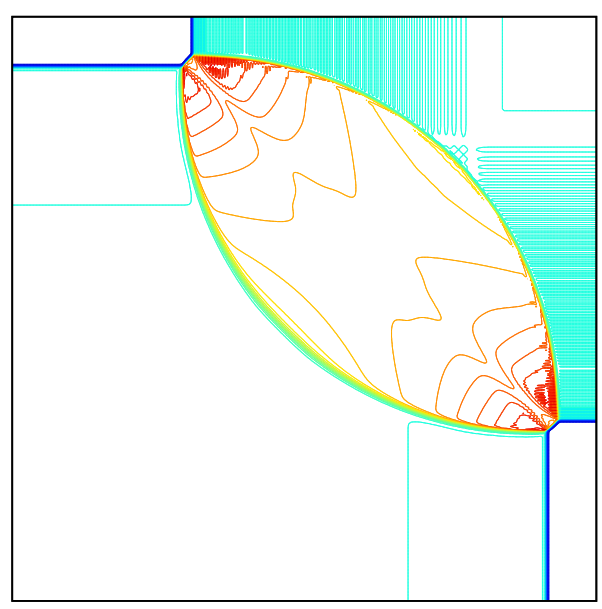

Density-Upwind scheme

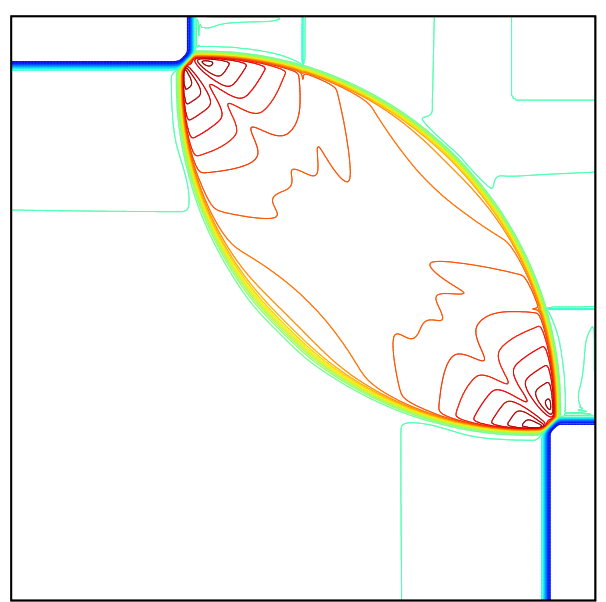

Density-Upwind scheme with WLR viscosity

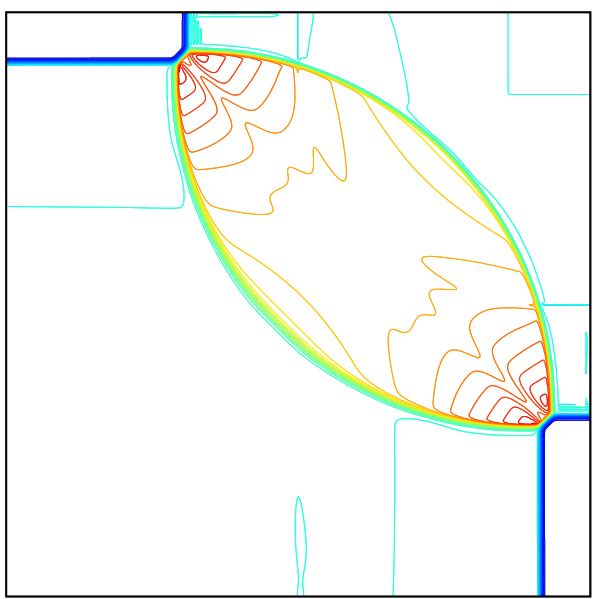

Density-Upwind scheme with entropic viscosity

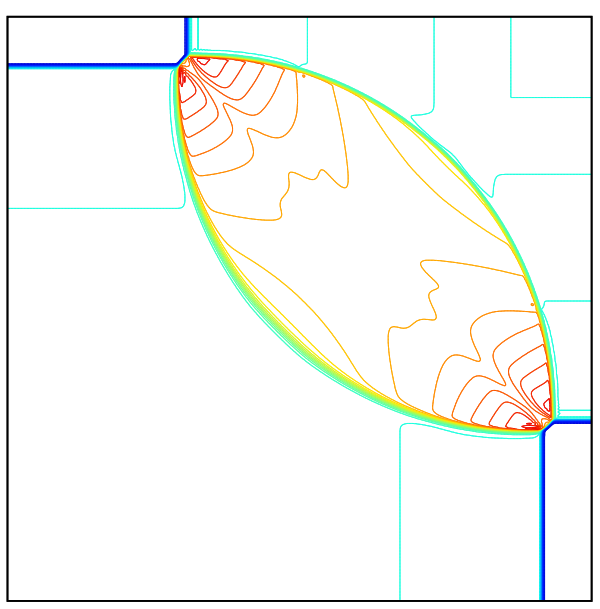

Density-Upwind scheme with constant viscosity

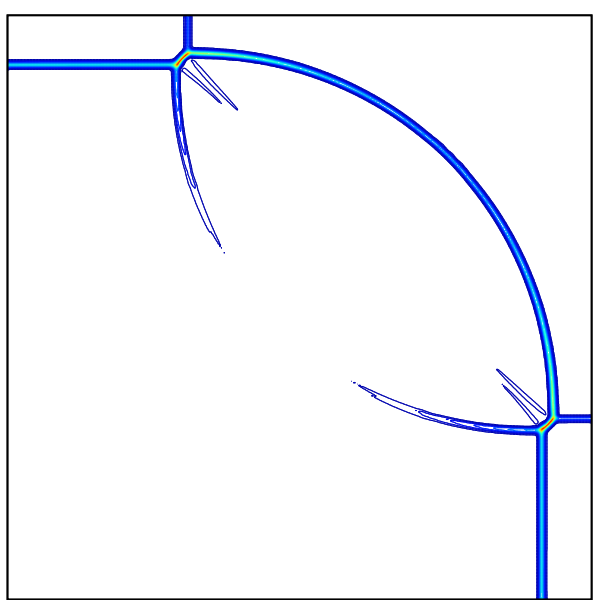

WLR viscosity

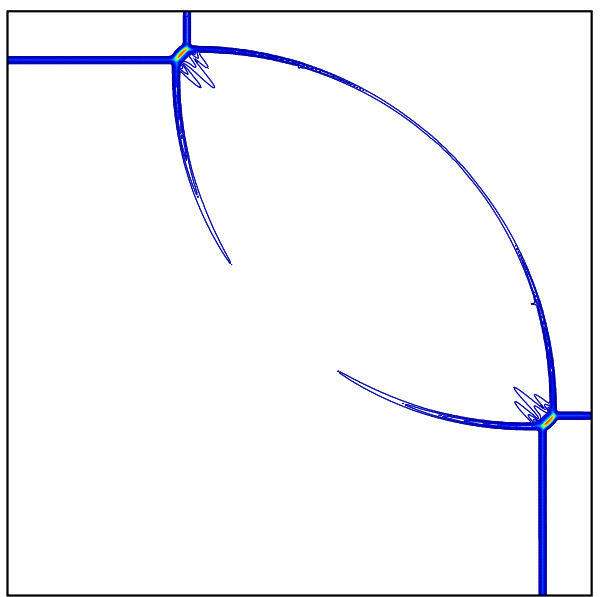

Entropic viscosity

Fig. 4.9. A two-dimensional Riemann problem: Configuration 4 in [30] $h=1 / 400$ and $\delta t=h / 10-$ Results at $t=0.3$. 
4.4. The Mach $=3$ wind tunnel with a step. This benchmark has been popularized in [43]. The computational domain is $\Omega=\bar{\Omega} \backslash \mathcal{S}$, with $\bar{\Omega}=(0,3) \times(0,1)$, and $\mathcal{S}=(0.6,3) \times(0,0.2)$. The time interval is $(0,4)$. A Mach 3 flow is coming from the left boundary $\{0\} \times(0,1)$ with the following properties:

$$
\left[\begin{array}{l}
\rho \\
\boldsymbol{u} \\
p
\end{array}\right]\left(\left(0, x_{2}\right)^{t}, t\right)=\left[\begin{array}{c}
1.4 \\
(3,0)^{t} \\
1
\end{array}\right], \quad \forall x_{2} \in(0,1), \forall t \in(0,4) .
$$

The initial data is the same as the inflow conditions:

$$
\left[\begin{array}{l}
\rho \\
\boldsymbol{u} \\
p
\end{array}\right](\boldsymbol{x}, 0)=\left[\begin{array}{c}
1.4 \\
(3,0)^{t} \\
1
\end{array}\right], \quad \forall \boldsymbol{x} \in \Omega .
$$

The right boundary $\{3\} \times(0,1)$ is free, since the flow leaves the domain at a velocity greater than the sound speed. Finally we prescribe a wall condition $(\boldsymbol{u} \cdot \boldsymbol{n}=0$, where $\boldsymbol{n}$ is the unit outward normal on $\partial \Omega$ ) on the rest of the boundary.

We display on figure 4.10 the results obtained at $t=4$ with the MAC space discretization, using the MUSCL interpolation. The mesh is a $4800 \times 1600$ uniform grid from which we remove the cells included in $\mathcal{S}$. The time step is set to $\delta t=$ $h / 10=6.2510^{-5}$, which corresponds to a CFL number approximatively equal to 0.5 with respect to the celerity of the fastest wave (equal to 4 at the inlet boundary). The artificial diffusion coefficient is $\nu=0.001$ (which is very weak, since the range of the diffusion induced by a first order upwinding is $\rho\|\boldsymbol{u}\| h / 2=\nu_{u p} h$ with $\nu_{u p} \sim 2$ ). Results are comparable to those presented in the recent literature (see e.g. $[13,22$, $44,12,16,15,2])$. The scheme seems to accurately capture discontinuities; however, the Kevin-Helmoltz instability which is often observed at the contact discontinuity issued from the Mach triple point is not present here, and adding some numerical diffusion (so artificial shear stresses) does not destabilize this slip line. A spurious Mach reflection at the bottom boundary is observed on coarser versions of the mesh, but is much attenuated here. 

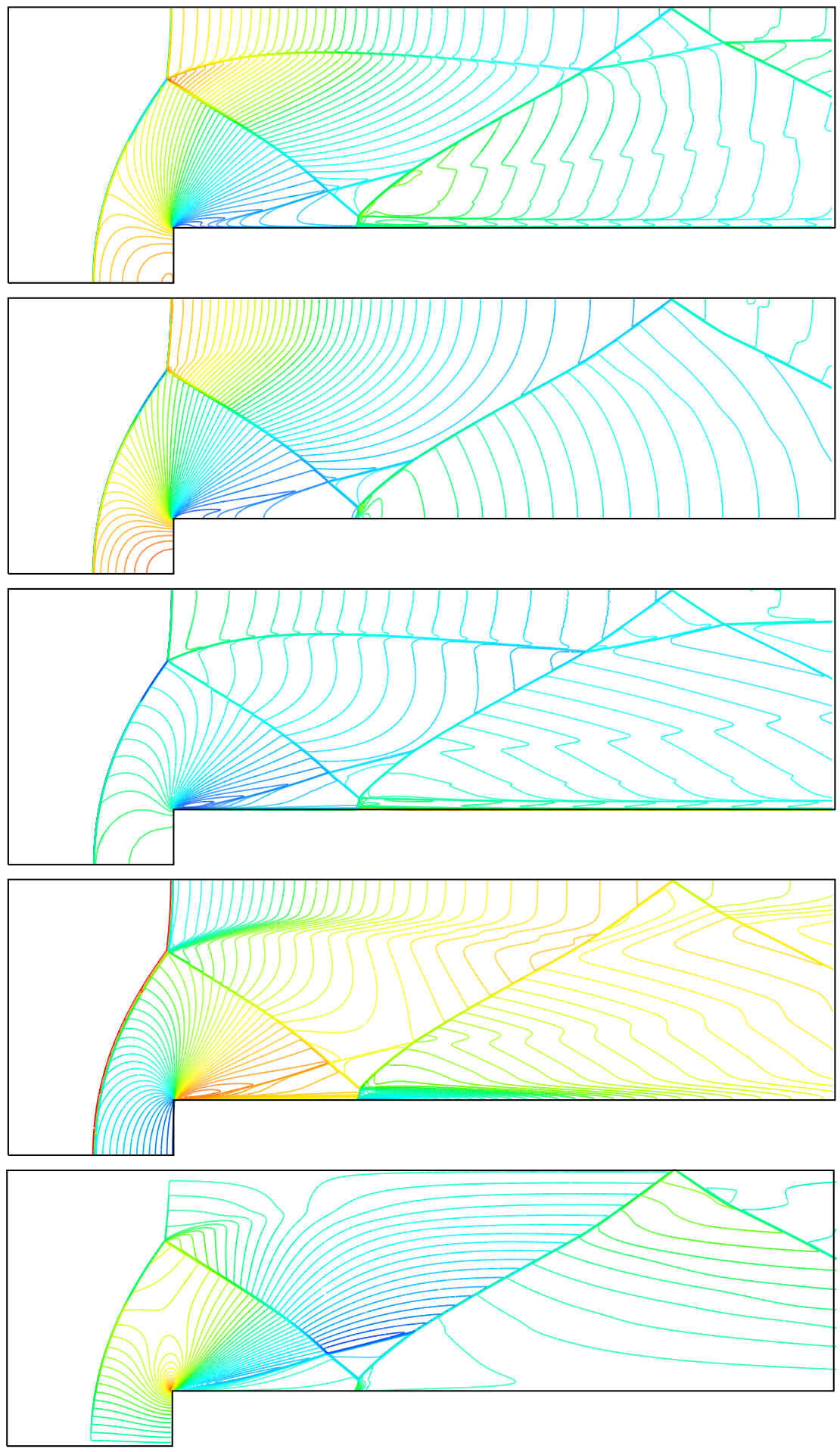

FIG. 4.10. Mach 3 step - From top to bottom: density, pressure, internal energy, first and second component of the velocity at $t=4$, obtained with $h=2.5 \times 10^{-3}, \delta t=10^{-3}$ and $\nu=10^{-3}$. The variation intervals of the unknowns are $\rho \in[0.235,6.4], p \in[0.216,12.04], \mathcal{H} \in[2.46,8.11]$, $u_{1} \in[0 ., 3.046]$, and $u_{2} \in[-0.92,1.82]$. 


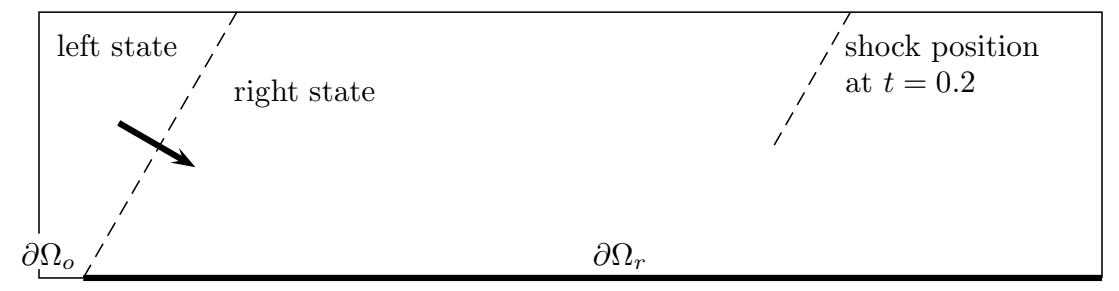

FIG. 4.11. Double Mach reflection - Geometry and initial conditions.

4.5. The double Mach reflection. This section is devoted to another classical test case, once again introduced in [43], which consists in a Mach=10 shock impacting a wall, the angle between the shock line and the wall being equal to $60^{\circ}$ (see Figure 4.11). The right state (pre-shock) initial conditions correspond to a fluid at rest, at the pressure $p_{R}=1$ and with $\rho_{R}=1.4$ (so the speed of sound is equal to 1 ). The left state is computed thanks to the Rankine-Hugoniot conditions, supposing that the shock velocity is equal to $\omega=10$ :

$$
\left[\begin{array}{l}
\rho_{R} \\
\boldsymbol{u}_{R} \\
p_{R}
\end{array}\right]=\left[\begin{array}{c}
1.4 \\
(0,0)^{t} \\
1
\end{array}\right], \quad\left[\begin{array}{l}
\rho_{L} \\
\boldsymbol{u}_{L} \\
p_{L}
\end{array}\right]=\left[\begin{array}{c}
8 \\
8.25(\sqrt{3} / 2,1 / 2)^{t} \\
116.5
\end{array}\right] .
$$

The computational domain is $\Omega=(0,4) \times(0,1)$, and we suppose that the wall lies in the bottom of the domain, more precisely $\partial \Omega_{w}=(1 / 6,4) \times\{0\}$. At $t=0$, the shock impinges the reflecting wall (at $x_{1}=1 / 6$ ), so the fluid is in the left state for $x_{1} \leq 1 / 6+x_{2} / \sqrt{3}$ and in the right state in the rest of the domain. Then, in the zones of $\Omega$ which are not perturbed by the reflections, the shock moves with a velocity equal to $\omega(\sqrt{3} / 2,-1 / 2)^{t}$. The external pressure at the outflow boundary $\partial \Omega_{o}$ is thus prescribed to $p_{L}=116.5$ at all time. On the top of the domain $(0,4) \times\{1\}$, the boundary condition is consistent to the undisturbed shock wave, thus the unknowns $\rho, \boldsymbol{u}$ and $p$ are prescribed to the left state values for $x_{1} \leq 1 / 6+1 / \sqrt{3}+(2 * \omega / \sqrt{3}) t$ and to the right state values on the other part of the boundary. Finally, on $\{4\} \times(0,1)$, the velocity is prescribed to $\boldsymbol{u}_{R}=(0,0)^{t}$.

We use a $1600 \times 400$ structured mesh, with the MAC space discretization. The time step is $\delta t=h / 100$ (which roughly corresponds to a CFL of $1 / 7$ with respect to the maximum wave celerity in the left state). The artificial stabilizing diffusion coefficient is set to $\nu=0.01$ (while the artificial diffusion which results from the upwinding scales as $\rho\|\boldsymbol{u}\| h / 2=\nu_{u p} h$ with $\left.\nu_{u p} \sim 30\right)$. The obtained fields at $t=0.2$ are shown on Figures 4.11. They comfort the previous observations: the approximate solution seems correct (see $[13,22,12,46,16,15,7]$ for other recents results), with rather sharp interfaces, while the slip lines are not subject to instabilities. This is confirmed by computations performed with refined meshes, dividing the cells by 2 in each direction (to obtain a $3200 \times 800$ structured mesh) and by $4(6400 \times 1600$ mesh); we give on Figure 4.13 the obtained internal energy field in the reflection structure. 

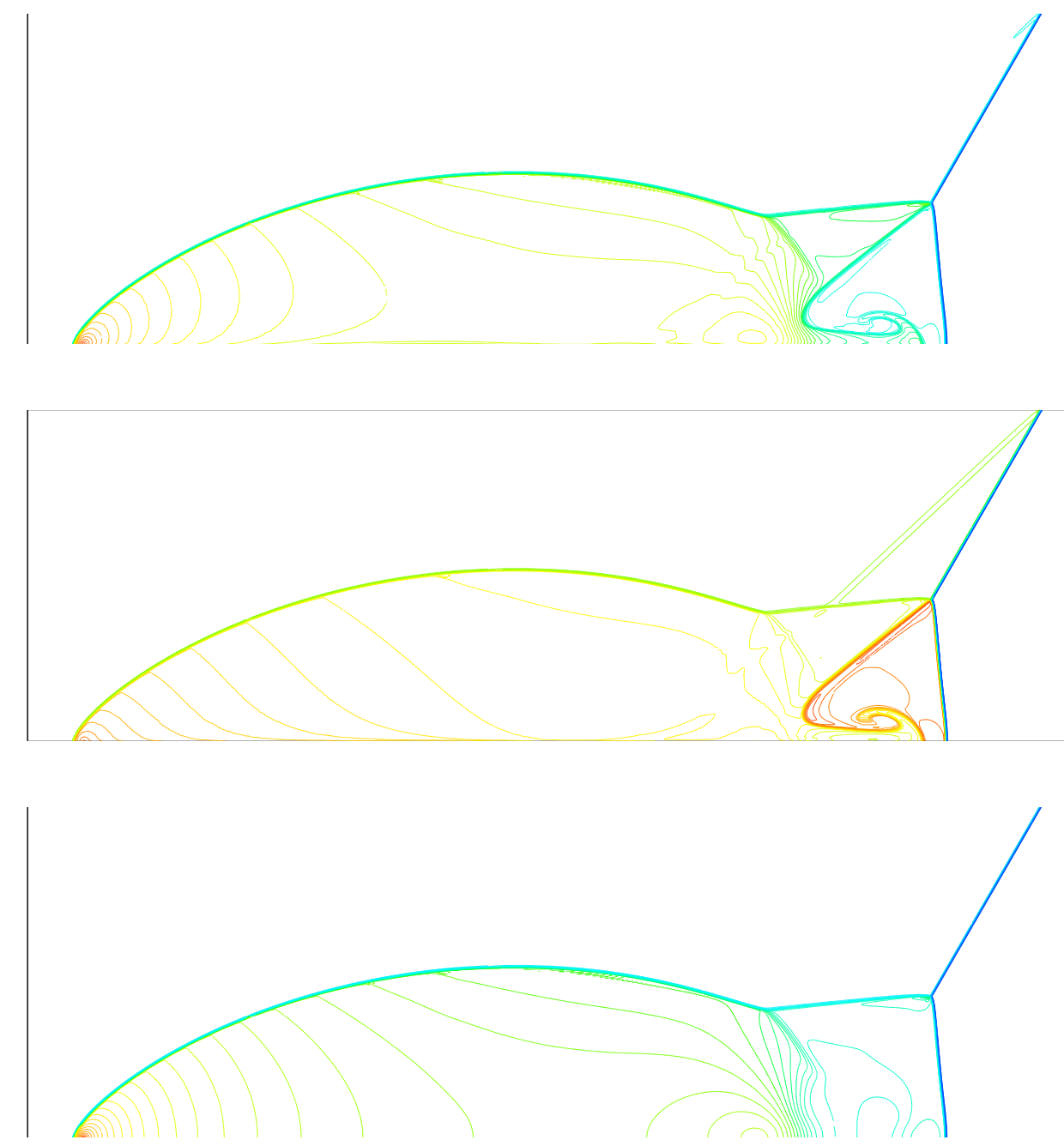

FIG. 4.12. Double Mach reflection - From top to bottom: density, internal energy and pressure at $t=0.2$, obtained with $h=2.510^{-3}, \delta t=2.510^{-5}$ and $\nu=0.01$. The variation ranges of the unknowns are $\rho \in[1.4,22.35], e \in[1.786,63.53]$ and $p \in[1,536]$. A right part of the domain, where the solution is constant, is not drawn. 

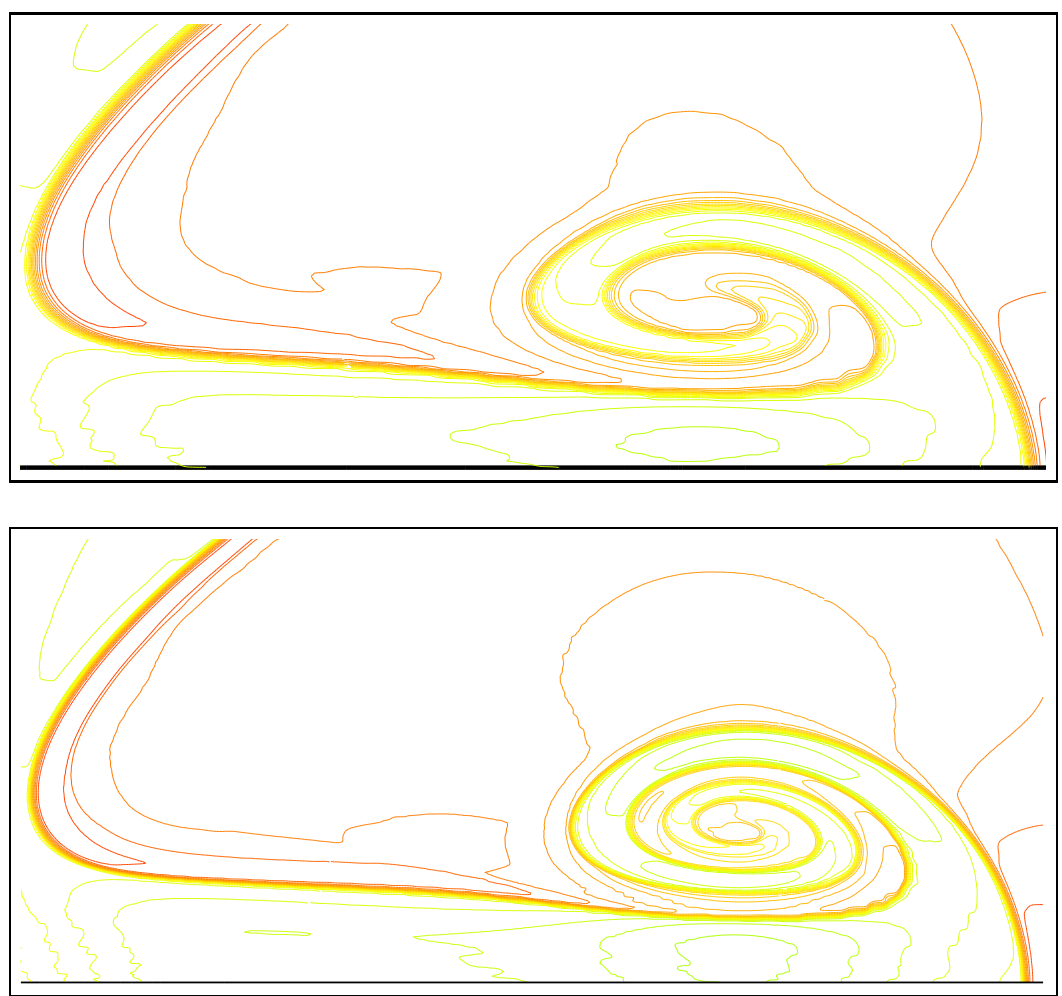

FIG. 4.13. Double Mach reflection, zoom of the internal energy isolines obtained with a $3200 \times$ 800 mesh (top) and with a $6400 \times 1600$ mesh (bottom).

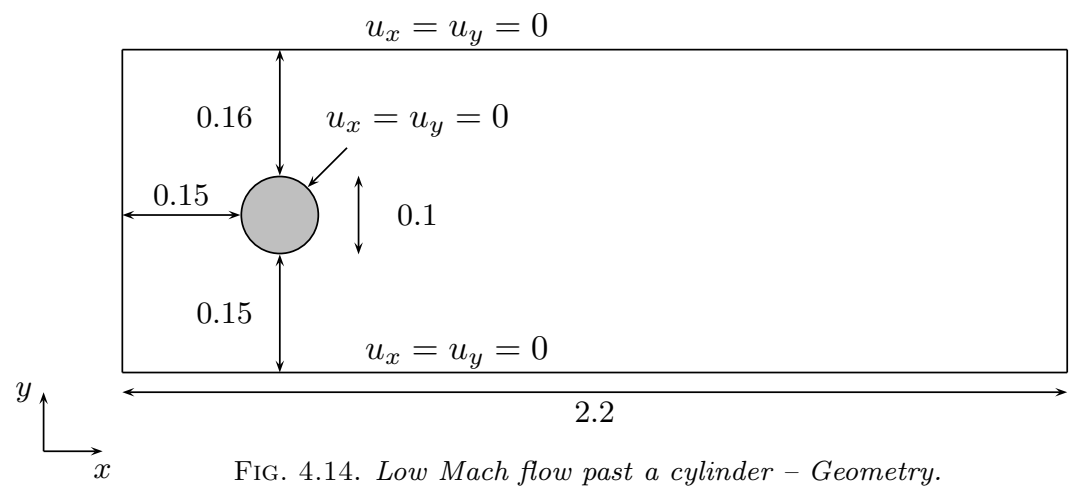

4.6. A $\mathbf{M a c h}=\mathbf{1 0}$ flow past a cylinder. The last test case is a compressible version of a benchmark originally developed for incompressible Navier-Stokes solvers [36]. The geometry of the problem is described in Figure 4.14. The fluid enters the domain on the left boundary with a constant velocity:

$$
\boldsymbol{u}=(1,0)^{t} .
$$

The Mach number of the flow entering the domain is chosen to be equal to 10 , so $c=(\gamma p / \rho)^{1 / 2}=0.1$, which is realized with:

$$
\left[\begin{array}{l}
\rho \\
p
\end{array}\right]=\left[\begin{array}{c}
1.0 \\
1 / 140
\end{array}\right]
$$

Initial conditions are the same as inlet values. The right boundary condition is free, and we impose a wall condition on the cylinder, the top and the bottom boundaries. The computationnal time interval is set to $(0,5)$. 


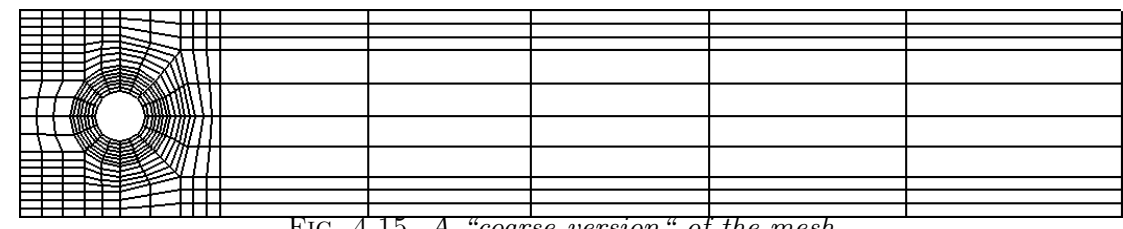

FIG. 4.15. A "coarse version" of the mesh.
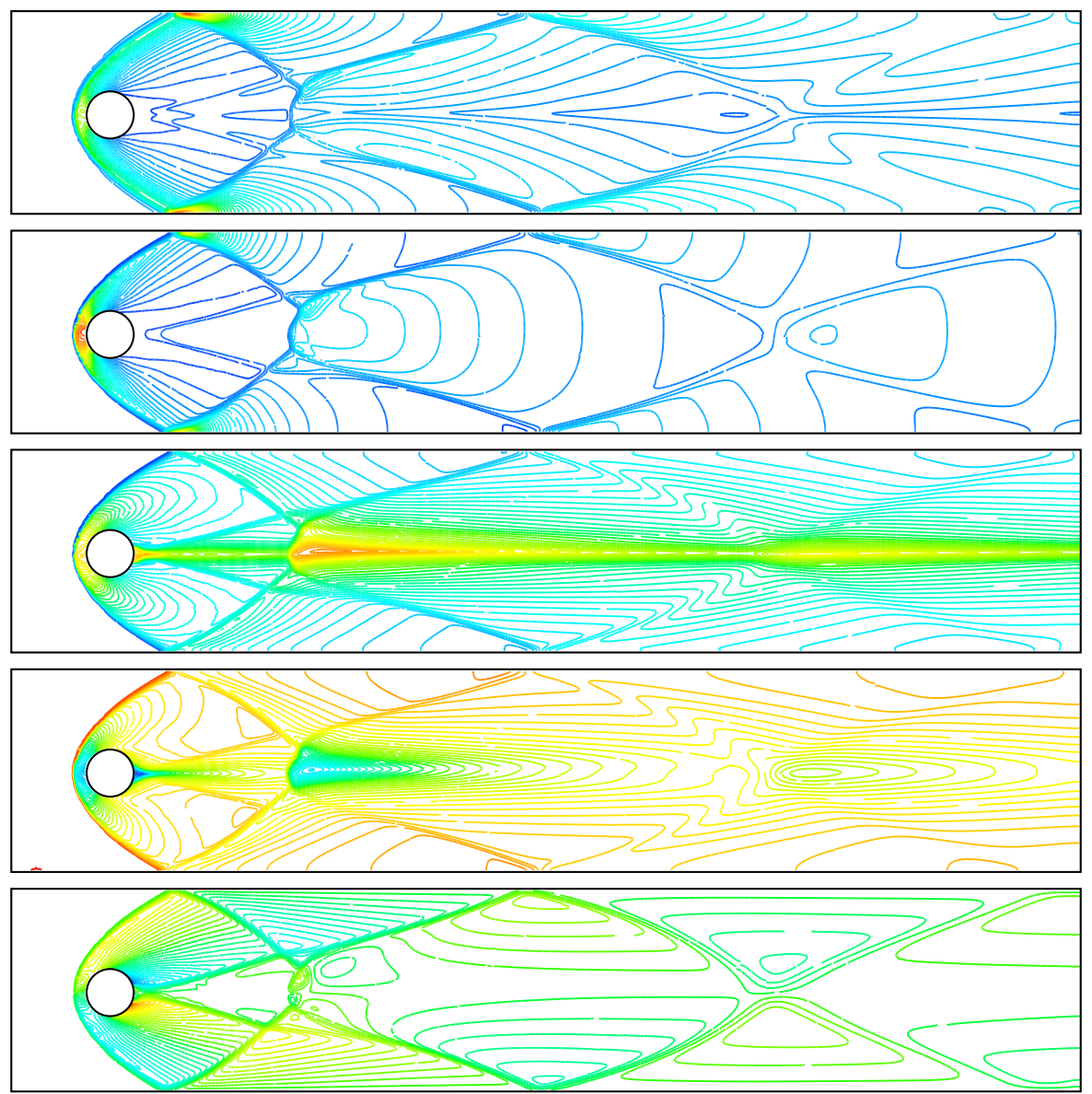

FIG. 4.16. Mach=10 flow past a cylinder - From top to bottom: density, pressure, internal energy, $x$-component of velocity and $y$-component of velocity at $t=5$. The variation ranges of the unknowns are $\rho \in[0.804,12.23], p \in[0.002,1.219], e \in[0.178,0.536], u_{1} \in[-0.11,1]$, and the value $u_{1}=0$ corresponds to the fourth iso-line, $u_{2} \in[-0.326,0.327]$.

The space approximation is performed with the RT discretization. A coarse version of the meshes used for this computation is presented in Figure 4.15; refined versions of this mesh are obtained by reducing the space step along the characteristic lines (the boundaries and the circles around the cylinder). We choose a stabilization coefficient $\nu \simeq 0.05$ which roughly corresponds to $1 / 10$ of the upwind dissipation (therefore much greater than the stabilization coefficient used with structured discretizations), and seems to be the lowest possible value (for $\nu \simeq 0.01$, strong distortions of the shock upstream the obstacle appear). The time step is set to $\delta t=10^{-4}$ and the computations are performed on a mesh with $5.3110^{5}$ cells which corresponds approximately to a space step of $h=0.001$. The speed of the fastest wave being 1.1, the acoustic CFL is close to 0.1 .

We present in Figure 4.16 the results obtained at $t=5$. We observe a strong shock in front of the cylinder. Subsequent weak shock reflections yield an X-structure for the pressure and density fields, which is progressively damped by the scheme diffusion. 
5. Conclusion. In this paper we presented a decoupled scheme based on staggered meshes for the Euler equations, using a MUSCL-like interpolation, which is a higher order version of the decoupled upwind scheme presented in [27]. This algorithm uses a quasi second-order monotone strategy which consists, equation by equation, in implementing a MUSCL type discretization with respect to the material velocity of the convection term. The pressure gradient is defined as the transpose of the natural velocity divergence, and is thus centered. As in [27], the scheme solves the internal energy balance instead of the total energy balance, to ensure the positivity of the internal energy thanks to the above-mentioned monotone technique; because of the staggered nature of the scheme, the total energy balance is only recovered at the limit of vanishing time and space steps, thanks to the addition of corrective source terms in the discrete internal energy balance.

Under CFL-like conditions which are based on the material velocity only, this scheme preserves the positivity of the density, the pressure and of the internal energy (in other words, the scheme preserves the convex set of admissible states). However, numerical studies show that the largest time step before blow-up is smaller than suggested by this condition. This behaviour was to be expected, since this condition only involves the velocity (and not the celerity of the acoustic waves): indeed, if it were the only limitation, we would obtain an explicit scheme stable up to the incompressible limit. However, the mechanisms leading to the blow-up of the scheme (or, conversely, the way to fix the time step to ensure stability) remain to be clarified, even if one may anticipate from qualitative arguments (the scheme should allow a "transport of the information" at the same speed as the continuous problem) that the time step should be small enough to avoid that the waves cross more than one cell per time step.

On going work is devoted to show that the scheme is consistent, in the sense that, if a sequence of numerical solutions obtained with increasingly refined meshes (and, accordingly, decreasing time steps) converges, then the limit is a weak solution to the continuous problem. Moreover, the scheme has been shown to satisfy some entropy inequalities [17], so that the limits of convergent sequences may be shown to be entropy solutions.

Another point of further investigation concerns the design of a discretization scheme that would be able to cope with non- conforming locally refined meshes. This work is now being undertaken.

Since the proposed scheme uses very simple numerical fluxes, it is well suited to large multi-dimensional parallel computing applications, and such studies are indeed performed at IRSN. Still for the same reasons (and, in particular, because the construction of the discretization does not require the solution of the Riemann problem), it seems that the presented approach offers natural extensions to more complex problems, such as reacting flows; this is under development at IRSN, for applications to explosion hazards.

Appendix A. Heun-like schemes and a toy ODE system.

Let us consider the following system of Ordinary Differential Equations:

$$
\begin{aligned}
& u^{\prime}+u=0, \\
& v^{\prime}+u=0 .
\end{aligned}
$$

The standard first order explicit scheme for this system reads:

$$
\begin{aligned}
& u^{n+1}=u^{n}-\delta t u^{n}, \\
& v^{n+1}=v^{n}-\delta t u^{n} .
\end{aligned}
$$

Let us build a Heun-like scheme by the following procedure: first perform a first order explicit step to obtain a tentative solution $\left(u^{n+\frac{1}{3}}, v^{n+\frac{1}{3}}\right)$, then perform a first order explicit step with initial solution $\left(u^{n+\frac{1}{3}}, v^{n+\frac{1}{3}}\right)$ to obtain a second tentative solution 
$\left(u^{n+\frac{2}{3}}, v^{n+\frac{2}{3}}\right)$ and finally compute $\left(u^{n+1}, v^{n+1}\right)$ as the mean value of $\left(u^{n}, v^{n}\right)$ and $\left(u^{n+\frac{2}{3}}, v^{n+\frac{2}{3}}\right)$. We thus obtain (for the unknown $v$ ) the following computation:

$$
\begin{aligned}
& u^{n+\frac{1}{3}}=u^{n}-\delta t u^{n}, \\
& v^{n+\frac{1}{3}}=v^{n}-\delta t u^{n}, \\
& v^{n+\frac{2}{3}}=v^{n+\frac{1}{3}}-\delta t u^{n+\frac{1}{3}}, \\
& v^{n+1}=\frac{1}{2}\left(v^{n}+v^{n+\frac{2}{3}}\right) .
\end{aligned}
$$

Using in (A.2d) the relation (A.2c), we get:

$$
v^{n+1}=\frac{1}{2}\left(v^{n}+v^{n+\frac{1}{3}}-\delta t u^{n+\frac{1}{3}}\right) .
$$

Then, using Equations (A.2a) and (A.2b) to express $u^{n+\frac{1}{3}}$ and $v^{n+\frac{1}{3}}$ respectively in this relation, we obtain:

$$
v^{n+1}=\frac{1}{2}\left(v^{n}+v^{n}-\delta t u^{n}-\delta t\left(u^{n}-\delta t u^{n}\right)\right)=v^{n}-\delta t u^{n}+\frac{\delta t^{2}}{2} u^{n} .
$$

Since, deriving Equation (A.1a), we obtain $u^{\prime \prime}=-u^{\prime}$ and thus, invoking once again (A.1a), $u^{\prime \prime}=u$, this last relation may be identified to an expression of $v^{n+1}$ thanks to a second order Taylor development from the solution at level $n$. We have thus built a second order scheme.

Let us now consider the following first order scheme, which differs from the scheme considered before by the fact that $u$ is taken at level $n+1$ in the second relation (which still yields a scheme with explicit steps, in the sense that the right-hand side is known at each step):

$$
\begin{aligned}
& u^{n+1}=u^{n}-\delta t u^{n}, \\
& v^{n+1}=v^{n}-\delta t u^{n+1} .
\end{aligned}
$$

The same procedure to derive a Heun-like scheme yields:

$$
\begin{aligned}
& u^{n+\frac{1}{3}}=u^{n}-\delta t u^{n}, \\
& v^{n+\frac{1}{3}}=v^{n}-\delta t u^{n+\frac{1}{3}}, \\
& u^{n+\frac{2}{3}}=u^{n+\frac{1}{3}}-\delta t u^{n+\frac{1}{3}}, \\
& v^{n+\frac{2}{3}}=v^{n+\frac{1}{3}}-\delta t u^{n+\frac{2}{3}}, \\
& v^{n+1}=\frac{1}{2}\left(v^{n}+v^{n+\frac{2}{3}}\right) .
\end{aligned}
$$

By the same computation as before, we obtain:

$$
v^{n+1}=v^{n}-\delta t u^{n}+\frac{3 \delta t^{2}}{2} u^{n}-\frac{\delta t^{3}}{2} u^{n} .
$$

The scheme is now only a first order one, which suggests that the Heun scheme must be built from the standard first order explicit discretization.

\section{REFERENCES}

[1] G. Ansanay-Alex, F. Babik, J.-C. Latché, and D. Vola. An L ${ }^{2}$-stable approximation of the Navier-Stokes convection operator for low-order non-conforming finite elements. International Journal for Numerical Methods in Fluids, 66:555-580, 2011.

[2] D. Balsara, S. Garain, and C.-W. Shu. An efficient class of WENO schemes with adaptive orders. Journal of Computational Physics, 326:780-804, 2016. 
[3] T. Barth and M. Ohlberger. Finite volume methods: foundation and analysis. In E. Stein, R. de Borst, and T. Hughes, editors, Encyclopedia of Computational Mechanics, Volume I, chapter 15. John Wiley \& Sons, 2004.

[4] F. Berthelin, T. Goudon, and S. Minjeaud. Kinetic schemes on staggered grids for barotropic Euler models: entropy-stability analysis. Mathematics of Computation, 84:2221-2262, 2015.

[5] C. Berthon, Y. Coudière, and V. Desveaux. Second-order MUSCL schemes based on Dual Mesh Gradient Reconstruction (DMGR). Mathematical Modelling and Numerical Analysis, 48:583-602, 2014.

[6] F. Bouchut. Nonlinear Stability of finite volume methods for hyperbolic conservation laws. Birkhauser, 2004.

[7] P. Buchmüller, J. Dreher, and C. Helzel. Finite volume WENO methods for hyperbolic conservation laws on Cartesian grids with adaptive mesh refinement. Applied Mathematics and Computation, 272:460-478, 2016.

[8] T. Buffard and S. Clain. Monoslope and multislope MUSCL methods for unstructured meshes. Journal of Computational Physics, 229:3745-3776, 2010.

[9] C. Calgaro, E. Chane-Kane, E. Creusé, and T. Goudon. $L^{\infty}$-stability of vertex-based MUSCL finite volume schemes on unstructured grids: Simulation of incompressible flows with high density ratios. Journal of Computational Physics, 229:6027-6046, 2010.

[10] $\mathrm{CALIF}^{3} \mathrm{~S}$. A software components library for the computation of reactive turbulent flows. https://gforge.irsn.fr/gf/project/isis.

[11] P. G. Ciarlet. Basic error estimates for elliptic problems. In P. Ciarlet and J. Lions, editors, Handbook of Numerical Analysis, Volume II, pages 17-351. North Holland, 1991.

[12] S. Clain, S. Diot, and R. Loubère. A high-order finite volume method for systems of conservation laws - Multi-dimensional Optimal Order Detection (MOOD). Journal of Computational Physics, 230:4028-4050, 2011.

[13] B. Cockburn and C.-W. Shu. The Runge-Kutta Discontinuous Galerkin method for conservation laws V - multidimensional systems. Journal of Computational Physics, 141:199-224, 1998.

[14] M. Crouzeix and P. Raviart. Conforming and nonconforming finite element methods for solving the stationary Stokes equations. RAIRO Série Rouge, 7:33-75, 1973.

[15] M. Dumbser and R. Loubère. A simple robust and accurate a posteriori sub-cell finite volume limiter for the discontinuous Galerkin method on unstructured meshes. Journal of Computational Physics, 319:163-199, 2016.

[16] M. Dumbser, O. Zanotti, A. Hidalgo, and D. Balsara. ADER-WENO finite volume scheme with space-time adaptive mesh refinement. Journal of Computational Physics, 248:257286, 2013.

[17] T. Gallouët, R. Herbin, J.-C. Latché, and N. Therme. Entropy estimates for a class of schemes for the Euler equations. submitted, 2018.

[18] L. Gastaldo, R. Herbin, W. Kheriji, C. Lapuerta, and J.-C. Latché. Staggered discretizations, pressure correction schemes and all speed barotropic flows. In Finite Volumes for Complex Applications VI - Problems and Perspectives - Prague, Czech Republic, volume 2, pages 39-56, 2011.

[19] E. Godlewski and P.-A. Raviart. Numerical approximation of hyperbolic systems of conservation laws. Springer, 1996.

[20] D. Grapsas, R. Herbin, W. Kheriji, and J.-C. Latché. An unconditionally stable staggered pressure correction scheme for the compressible Navier-Stokes equations. SMAI-JCM, 2:51-97, 2016.

[21] J.-L. Guermond and R. Pasquetti. Entropy-based nonlinear viscosity for Fourier approximations of conservation laws. Comptes Rendus de l'Académie des Sciences de Paris - Série I-Analyse Numérique, 346:801-806, 2008.

[22] J.-L. Guermond, R. Pasquetti, and B. Popov. Entropy viscosity method for nonlinear conservation laws. Journal of Computational Physics, 230:4248-4267, 2011.

[23] F. Harlow and A. Amsden. A numerical fluid dynamics calculation method for all flow speeds. Journal of Computational Physics, 8:197-213, 1971.

[24] F. Harlow and J. Welsh. Numerical calculation of time-dependent viscous incompressible flow of fluid with free surface. Physics of Fluids, 8:2182-2189, 1965.

[25] R. Herbin, W. Kheriji, and J.-C. Latché. On some implicit and semi-implicit staggered schemes for the shallow water and Euler equations. Mathematical Modelling and Numerical Analysis, 48:1807-1857, 2014.

[26] R. Herbin and J.-C. Latché. Kinetic energy control in the MAC discretization of the compressible Navier-Stokes equations. International Journal of Finites Volumes, 7, 2010.

[27] R. Herbin, J.-C. Latché, and T. Nguyen. Consistent segregated staggered schemes with explicit steps for the isentropic and full Euler equations. Mathematical Modelling and Numerical Analysis, to appear.

[28] A. Kurganov and Y. Liu. New adaptative artificial viscosity method for hyperbolic systems of conservation laws. Journal of Computational Physics, 231:8114-8132, 2012.

[29] A. Kurganov and E. Tadmor. Solution of two-dimensional Riemann problems for gas dynamics without Riemann problem solvers. Numerical Methods for Partial Differential Equations, 18:584-608, 2002.

[30] P. Lax and X.-D. Liu. Solution of two dimensional riemann problem of gas dynamics by positive schemes. SIAM Journal on Scientific Computing, 19:319-340, 1998.

[31] C. Le Touze, A. Murrone, and H. Guillard. Multislope MUSCL method for general unstructured 
meshes. Journal of Computational Physics, 284:389-418, 2015.

[32] M.-S. Liou. A sequel to AUSM, part II: AUSM+-up. Journal of Computational Physics, 214:137-170, 2006.

[33] M.-S. Liou and C. Steffen. A new flux splitting scheme. Journal of Computational Physics, 107:23-39, 1993.

[34] L. Piar, F. Babik, R. Herbin, and J.-C. Latché. A formally second order cell centered scheme for convection-diffusion equations on general grids. International Journal for Numerical Methods in Fluids, 71:873-890, 2013.

[35] R. Rannacher and S. Turek. Simple nonconforming quadrilateral Stokes element. Numerical Methods for Partial Differential Equations, 8:97-111, 1992.

[36] M. Schäfer and S. Turek. Benchmark Computations of Laminar Flow Around a Cylinder, volume 52 of Notes on Numerical Fluid Mechanics. 1996.

[37] C. Schulz-Rinne, J. Collins, and H. Glaz. Numerical solution of the Riemann problem for two-dimensional gas dynamics. SIAM Journal on Scientific Computing, 14:1394-1414, 1993.

[38] J. Steger and R. Warming. Flux vector splitting of the inviscid gaz dynamics equations with applications to finite difference methods. Journal of Computational Physics, 40:263-293, 1981.

[39] E. Toro. Riemann solvers and numerical methods for fluid dynamics - A practical introduction (third edition). Springer, 2009.

[40] E. Toro and M. Vázquez-Cendón. Flux splitting schemes for the Euler equations. Computers E Fluids, 70:1-12, 2012.

[41] B. Van Leer. Towards the ultimate conservative difference scheme, v,. J. Comput. Phys., 32:101-136, 1979

[42] B. Van Leer. Upwind and high-resolution methods for compressible flow: From donor cell to residual-distribution schemes. Comm. Comput. Physics, 1(2):192-206, 2006.

[43] P. Woodward and P. Colella. The numerical simulation of two-dimensional fluid flow with strong shocks. Journal of Computational Physics, 54:115-173, 1984.

[44] Z. Xu, Y. Liu, H. Du, G. Lin, and C.-W. Shu. Point-wise hierarchical reconstruction for discontinuous Galerkin and finite volume methods for solving conservation laws. Journal of Computational Physics, 230:6843-6865, 2011.

[45] G.-C. Zha and E. Bilgen. Numerical solution of Euler equations by a new flux vector splitting scheme. International Journal for Numerical Methods in Fluids, 17:115-144, 1993.

[46] X. Zhong and C.-W. Shu. A simple weighted essentially nonoscillatory limiter for Runge-Kutta discontinuous Galerkin methods. Journal of Computational Physics, 232:397-415, 2013. 